\title{
Unusual plastic deformation and damage features in titanium: Experimental tests and constitutive modeling
}

\author{
Benoit Revil-Baudard ${ }^{\mathrm{a}}$, Oana Cazacu ${ }^{\mathrm{a}, *}$, Philip Flater ${ }^{\mathrm{a}, \mathrm{b}}$, Nitin Chandola ${ }^{\mathrm{a}}$, \\ J.L. Alves ${ }^{\mathrm{c}}$ \\ a Department of Mechanical and Aerospace Engineering, University of Florida, REEF, 1350 N. Poquito Rd., Shalimar, FL, USA \\ ${ }^{\mathrm{b}}$ Air Force Research Laboratory, Eglin, FL, USA \\ ${ }^{\text {c } M E M S ~-~ M i c r o e l e c t r o m e c h a n i c a l ~ S y s t e m s ~ R e s e a r c h ~ U n i t, ~ D e p a r t m e n t ~ o f ~ M e c h a n i c a l ~ E n g i n e e r i n g, ~ U n i v e r s i t y ~ o f ~ M i n h o, ~ P o r t u g a l ~}$
}

\section{A R T I C L E I N F O}

\section{Article history:}

Received 18 May 2015

Received in revised form

24 December 2015

Accepted 7 January 2016

Available online 8 January 2016

\section{Keywords:}

$\alpha$-titanium

In-situ XCMT

Plasticity

Damage

Constitutive modeling

\begin{abstract}
A B S T R A C T
In this paper, we present an experimental study on plastic deformation and damage of polycrystalline pure HCP Ti, as well as modeling of the observed behavior. Mechanical characterization data were conducted, which indicate that the material is orthotropic and displays tension-compression asymmetry. The ex-situ and in-situ X-ray tomography measurements conducted reveal that damage distribution and evolution in this HCP Ti material is markedly different than in a typical FCC material such as copper. Stewart and Cazacu (2011) anisotropic elastic/plastic damage model is used to describe the behavior. All the parameters involved in this model have a clear physical significance, being related to plastic properties, and are determined from very few simple mechanical tests. It is shown that this model predicts correctly the anisotropy in plastic deformation, and its strong influence on damage distribution and damage accumulation. Specifically, for a smooth axisymmetric specimen subject to uniaxial tension, damage initiates at the center of the specimen, and is diffuse; the level of damage close to failure being very low. On the other hand, for a notched specimen subject to the same loading the model predicts that damage initiates at the outer surface of the specimen, and further grows from the outer surface to the center of the specimen, which corroborates with the in-situ tomography data.
\end{abstract}

(c) 2016 Elsevier Ltd. All rights reserved.

\section{Introduction}

Titanium materials that are predominantly hexagonal close-packed (hcp) are known to display at room temperature plastic anisotropy and a strong tension-compression asymmetry (e.g. for high-purity hcp - Ti, see data reported by Nixon et al. (2010a, 2010b); for TiAl6V, see data of Gilles et al. (2011)). This tension-compression asymmetry at macroscopic level is a consequence of the operation of specific crystallographic deformation mechanisms. Concerning the identification of crystallographic deformation mechanisms operational in high-purity Ti, the reader is referred to the studies of Salem et al. (2003), Knezevic et al. (2013) for monotonic uniaxial loadings, Bouvier et al. (2012) for simple shear monotonic, and cyclic loadings; concerning commercial-purity Ti, see for e.g. Chun et al. (2005), Benhenni et al. (2013), etc. As concerns modeling the mechanical behavior at macroscopic level, it is generally agreed that classical plasticity models such as von Mises or Hill

\footnotetext{
* Corresponding author.

E-mail address: cazacu@reef.ufl.edu (O. Cazacu).
} 
(1948) cannot capture the specificities of the plastic deformation in Ti materials (e.g. Kuwabara et al. (2001), Nixon et al. (2010a)). As pointed out in the review of Banerjee and Williams (2013), recently developed macroscopic level elastic/plastic constitutive models (e.g. Cazacu and Barlat, 2004, Cazacu et al. 2006, Nixon et al. 2010a) are able to capture the main features of the plastic deformation of hcp-titanium. Specifically, the capabilities of these models to predict both the anisotropy and tension-compression asymmetry in plastic deformation have been demonstrated for both low-strain rates loadings (e.g. for pure Ti under uniaxial tension, uniaxial compression, bending, see Nixon et al., 2010 b; under torsion, see Revil-Baudard et al., 2014; for TiAl6V under tension, compression, and plane-strain tension, see Gilles et al. 2013, etc.), and high-rate and impact loadings (e.g. Revil-Baudard et al., 2015).

Although titanium materials are textured, mechanical data (stress-strain and/or damage response) reported in the literature concern only one loading orientation (e.g. Huez et al., 1998). Moreover, the modeling of the room-temperature damage and failure of titanium materials is done using either empirical laws for the strain at failure, or evolution laws for the rate of void growth (e.g. Rice and Tracey (1969) law and its various modifications). However, Rice and Tracey (1969) void growth law or the most widely used criteria for ductile damage (e.g. Gurson (1977) and its various modifications) cannot realistically describe damage in Ti materials because the core hypothesis of such models is that the plastic behavior of the matrix is governed by the isotropic von Mises criterion, which is a criterion critically inadequate for Ti materials.

Using rigorous upscaling techniques based on Hill-Mandel lemma (Hill, 1967; Mandel, 1972), Stewart and Cazacu (2011) recently developed an analytic anisotropic model for porous polycrystals that accounts for the combined effects of tensioncompression asymmetry and evolving anisotropy on damage. The unusual properties of the yield loci of a porous hcp polycrystalline Zr-like material predicted with this model were validated by full-field crystal plasticity simulations (see Lebensohn and Cazacu, 2012).

In this paper, we present an experimental study on plastic deformation and damage of a polycrystalline pure Ti material, as well as modeling of the observed behavior. The Stewart and Cazacu (2011) model, which will be used for prediction of plastic deformation and damage evolution in this Ti material is briefly presented in Section 2. All model parameters are expressible in terms of a few coefficients related to the plastic properties of the material. Their identification is done based on a few simple uniaxial tension tests on flat specimens, and uniaxial compression tests on cylindrical specimens (see Sections 3.1-3.2). To further study damage in this Ti material, additional uniaxial tension tests on axisymmetric cylindrical specimens of circular cross-section were conducted. Both smooth and notched geometries were considered. X-ray microcomputed tomography (XCMT) measurements both ex-situ and in-situ were conducted. Comparison between data and finite-element (FE) predictions of both local and global deformation, and porosity evolution obtained with the model are presented (Section 4). We conclude with a summary of the main experimental findings, and conclusions concerning the capabilities of the model to capture the key features of plasticity-damage couplings in Ti (see Section 5).

\section{Elastic-plastic damage model}

The total rate of deformation $\mathbf{D}$ (the symmetric part of $\dot{F}^{-1}$ where $\mathbf{F}$ is the deformation gradient) is considered to be the sum of an elastic part and a plastic part $\mathbf{D}^{\mathrm{p}}$. The elastic response is described as

$$
\dot{\boldsymbol{\sigma}}=\mathbf{C}^{\mathrm{e}}:\left(\mathbf{D}-\mathbf{D}^{\mathrm{p}}\right)
$$

where $\boldsymbol{\sigma}$ is the Green-Naghdi derivative (see, Green and Naghdi, 1965, ABAQUS, 2009) of the Cauchy stress tensor $\boldsymbol{\sigma}, \mathbf{C}^{\mathrm{e}}$ is the fourth-order stiffness tensor while ":" denotes the doubled contracted product between the two tensors.

The plastic strain rate is defined with respect to the stress potential $\varphi$ as:

$$
\mathbf{D}^{\mathrm{p}}=\dot{\lambda} \frac{\partial \phi}{\partial \boldsymbol{\sigma}}
$$

where $\dot{\lambda}$ is the plastic multiplier. The plastic potential that will be used was derived by Stewart and Cazacu (2011). It has the following expression:

$$
\varphi(\sigma, f)=\hat{\mathrm{m}}^{2} \frac{\sum_{i=1}^{3}\left(\left|\hat{\sigma}_{\mathrm{i}}\right|-k \hat{\sigma}_{\mathrm{i}}\right)^{2}}{\bar{\sigma}_{\mathrm{x}}^{\mathrm{T}}}+\bar{\sigma}_{\mathrm{x}}^{\mathrm{T}}\left(2 f \cosh \left(\frac{3 \sigma_{\mathrm{m}}}{h \bar{\sigma}_{\mathrm{x}}^{\mathrm{T}}}\right)-\left(1+f^{2}\right)\right)=0,
$$

where $k$ is a parameter describing the tension-compression asymmetry of the matrix; $f$ is the void volume fraction, and $\hat{\sigma}_{1}, \hat{\sigma}_{2}, \hat{\sigma}_{3}$ are the principal values of the transformed stress tensor

$$
\widehat{\boldsymbol{\sigma}}=\mathrm{L}: \boldsymbol{\sigma}^{\prime} \text {. }
$$

In Eq. (4) $\boldsymbol{\sigma}^{\prime}$ is the deviator of the Cauchy stress tensor $\sigma,\left(\boldsymbol{\sigma}^{\prime}=\boldsymbol{\sigma}-\sigma_{\mathrm{m}} \mathbf{I}, \sigma_{\mathrm{m}}=(1 / 3) \boldsymbol{\sigma}\right.$ : I, with $\mathbf{I}$ being the second-order identity tensor), $\mathbf{L}$ is a fourth-order symmetric tensor describing the anisotropy of the matrix. Let $(\mathbf{x}, \mathbf{y}, \mathbf{z})$ be the reference frame associated with orthotropy. In the case of a plate, $\mathbf{x}, \mathbf{y}$ and $\mathbf{z}$ represent the rolling, transverse and normal directions, respectively. Relative to the orthotropy axes, the fourth-order tensor $\mathbf{L}$ is represented in Voigt notation by: 


$$
\mathbf{L}=\left[\begin{array}{llllll}
\mathrm{L}_{11} & \mathrm{~L}_{12} & \mathrm{~L}_{13} & & & \\
\mathrm{~L}_{12} & \mathrm{~L}_{22} & \mathrm{~L}_{23} & & & \\
\mathrm{~L}_{13} & \mathrm{~L}_{23} & \mathrm{~L}_{33} & & & \\
& & & \mathrm{~L}_{44} & & \\
& & & & \mathrm{~L}_{55} & \\
& & & & & \mathrm{~L}_{66}
\end{array}\right]
$$

In the expression of $\varphi(\sigma, f)$ given by Eq. (3), $\hat{\mathrm{m}}$ is a constant which depends on the anisotropy coefficients $\mathrm{L}_{i j}, i j,=1 \ldots 3$ (see Eq. (5)) and the strength differential parameter $k$, i.e.:

$$
\hat{\mathrm{m}}=\left[\left(\left|\Phi_{1}\right|-\mathrm{k} \Phi_{1}\right)^{2}+\left(\left|\Phi_{2}\right|-\mathrm{k} \Phi_{2}\right)^{2}+\left(\left|\Phi_{3}\right|-\mathrm{k} \Phi_{3}\right)^{2}\right]^{-1 / 2},
$$

where $\Phi_{1}=\left(2 \mathrm{~L}_{11}-L_{12}-L_{13}\right) / 3, \Phi_{2}=\left(2 \mathrm{~L}_{12}-L_{22}-L_{23}\right) / 3, \Phi_{3}=\left(2 \mathrm{~L}_{13}-L_{23}-L_{33}\right) / 3$. The parameter $\mathrm{h}$ in the potential $\varphi(\boldsymbol{\sigma}, f)$ controls damage evolution. It depends on the matrix anisotropy and the sign of the mean stress, $\sigma_{\mathrm{m}}$. Its expression is:

$$
\begin{aligned}
& \mathrm{h}=\sqrt{\mathrm{n}\left(4 \mathrm{t}_{1}+6 \mathrm{t}_{2}\right) / 5}, \\
& \mathrm{n}= \begin{cases}\frac{3}{\hat{\mathrm{m}}^{2}\left(3 \mathrm{k}^{2}-2 \mathrm{k}+3\right)} & \text { if } \sigma_{\mathrm{m}}<0, \\
\frac{3}{\hat{\mathrm{m}}^{2}\left(3 \mathrm{k}^{2}+2 \mathrm{k}+3\right)} & \text { if } \sigma_{\mathrm{m}} \geq 0 .\end{cases}
\end{aligned}
$$

The scalars $t_{1}$ and $t_{2}$ involved in the expression of h given by Eq. (7) are expressed as:

$$
\begin{aligned}
& \mathrm{t}_{1}=3\left(\mathrm{~B}_{13} \mathrm{~B}_{23}+\mathrm{B}_{12} \mathrm{~B}_{23}+\mathrm{B}_{12} \mathrm{~B}_{13}+2 \mathrm{~B}_{12}^{2}+2 \mathrm{~B}_{13}^{2}+2 \mathrm{~B}_{23}^{2}\right) \\
& \mathrm{t}_{2}=\mathrm{B}_{44}^{2}+\mathrm{B}_{55}^{2}+\mathrm{B}_{66}^{2}
\end{aligned}
$$

In the above equation, $B_{i j}$, with $i, j=1 \ldots 3$, are the components of the inverse of the tensor $\mathbf{L K}$, with $\mathbf{K}$ denoting the 4 th order deviatoric unit tensor,

$$
\mathbf{K}=\left[\begin{array}{ccccc}
2 / 3 & -1 / 3 & -1 / 3 & & \\
-1 / 3 & 2 / 3 & 1 / 3 & & \\
-1 / 3 & -1 / 3 & 2 / 3 & & \\
& & & 1 & \\
& & & & 1 \\
& & & & 1
\end{array}\right] .
$$

The expressions of the components of $\mathbf{B}$ in terms of the anisotropy coefficients $\mathrm{L}_{\mathrm{ij}}$ (i.e. components of the orthotropy tensor $\mathbf{L}$ ) are given in Appendix A.

Hardening of the matrix is considered to be governed by the effective plastic strain, $\bar{\varepsilon}^{\mathrm{p}}$. The rate of the effective plastic strain $\dot{\bar{\varepsilon}}^{\mathrm{p}}$ is obtained, assuming the equivalence of microscopic and macroscopic inelastic work and associated flow rule, as

$$
\bar{\sigma} \dot{\bar{\varepsilon}}^{\mathrm{p}}(1-f)=\boldsymbol{\sigma}: \mathbf{D}^{\mathrm{p}},
$$

so,

$$
\dot{\bar{\varepsilon}}^{\mathrm{p}}=\frac{\boldsymbol{\sigma}: \mathbf{D}^{\mathrm{p}}}{(1-f) \overline{\bar{\sigma}}}=\dot{\lambda} \quad \frac{\boldsymbol{\sigma}: \frac{\partial \phi}{\partial \boldsymbol{\sigma}}}{(1-f) \bar{\sigma}} .
$$

The rate of change of the void volume fraction $(\dot{f})$ is considered to result from the growth of existing voids and the nucleation of new ones. Void growth is obtained from mass conservation and the use of the plastic flow rule (Eq. (2)) in conjunction with Eq. (3). Void nucleation is considered to be due to plastic strain, as suggested by Gurson (1975) based on Gurland's (1972) experimental data, and to the mean stress, $\sigma_{\mathrm{m}}$, as discussed in Argon et al. (1975). Both plastic strain controlled nucleation and mean stress controlled nucleation are considered to follow a normal distribution with mean value $\left(\varepsilon_{N}, \sigma_{\mathrm{P}}\right)$ and a standard deviation $\left(s_{N}, \mathrm{~S}_{\mathrm{P}}\right)$, as proposed by Chu and Needleman (1980). Thus,

$$
\dot{\mathrm{f}}=(1-\mathrm{f}) \mathbf{D}^{\mathrm{p}}: \mathbf{I}+\mathrm{A}_{\mathrm{N}} \dot{\bar{\varepsilon}}^{\mathrm{p}}+\mathrm{B}_{\mathrm{N}} \dot{\sigma}_{\mathrm{m}},
$$


where

$$
\begin{aligned}
& A_{N}=\frac{f_{N}}{s_{N} \sqrt{2 \pi}} \exp \left[-\frac{1}{2}\left(\frac{\bar{\varepsilon}^{p}-\varepsilon_{N}}{s_{N}}\right)^{2}\right], \\
& B_{N}=\left\{\begin{array}{cc}
0 & \text { if } \dot{\sigma}_{m}<0 \\
\frac{f_{p}}{s_{P} \sqrt{2 \pi}} \exp \left[-\frac{1}{2}\left(\frac{\sigma_{m}-\sigma_{P}}{s_{P}}\right)^{2}\right] & \text { if } \dot{\sigma}_{m}>0
\end{array}\right.
\end{aligned}
$$

In the above equation, $f_{\mathrm{N}}$ and $f_{\mathrm{P}}$ represent the volume fraction of voids nucleated by the plastic strain, and by the mean stress, respectively. It is to be noted that in the absence of voids $(f=0)$, Stewart and Cazacu $(2011)$ criterion $\varphi(\sigma, f)$, given by Eq. (3), reduces to that of the matrix, i.e. the quadratic form of Cazacu et al. (2006) orthotropic criterion:

$$
\tilde{\sigma}_{e}=\sigma_{x}^{T},
$$

with

$$
\tilde{\sigma}_{e}=\hat{m} \sqrt{\sum_{i=1}^{3}\left(\left|\hat{\sigma}_{i}\right|-k \hat{\sigma}_{i}\right)^{2}}
$$

Note that the Cazacu et al. (2006) effective stress, $\tilde{\sigma}_{e}$, accounts for the tension-compression asymmetry of the matrix through the parameter $k$, and depends on all the invariants of the stress deviator, $\boldsymbol{\sigma}^{\prime}$, as well as on the mixed invariants of $\boldsymbol{\sigma}^{\prime}$ and the symmetry tensors associated with orthotropy, namely $\mathbf{M}_{1}=\mathbf{x} \otimes \mathbf{x}, \mathbf{M}_{2}=\mathbf{y} \otimes \mathbf{y}, \mathbf{M}_{3}=\mathbf{z} \otimes \mathbf{z}$ (see Cazacu et al., 2006). Thus, the anisotropic yield function $\varphi(\sigma, f)$ for the porous material given by Eq. (3) accounts for the combined effects of the matrix orthotropy and tension-compression asymmetry. Furthermore, since the parameter $h$ depends on the anisotropy coefficients $L_{i j}$, on the strength-differential parameter $k$, and on the sign of the applied mean stress $\sigma_{\mathrm{m}}($ see Eqs. (7)-(9) and (A.1), Stewart and Cazacu (2011) criterion given by Eq. (3) accounts for the combined effects of the tension-compression asymmetry and orthotropy on the dilatational response. In particular, according to this criterion, the yield surface of the porous solid does not display the centro-symmetry properties of the yield surface of a porous von Mises solid (see Cazacu et al. (2013)) or of a porous Tresca solid (see Cazacu et al. (2014)). Specifically, the yield limit for purely tensile hydrostatic loading is different than the yield limit for purely compressive hydrostatic loading. Indeed, according to the criterion, for tensile hydrostatic loading, yielding of the porous material occurs when $\sigma_{m}=\hat{p}_{Y}^{+}$, with

$$
\hat{\mathrm{p}}_{Y}^{+}=-\frac{\sigma_{x}^{T}}{3} \sqrt{\frac{3}{\hat{\mathrm{m}}^{2}\left(3 k^{2}+2 k+3\right)}\left(\frac{4 t_{1}+6 t_{2}}{5}\right)} \ln (f),
$$

whereas for compressive hydrostatic loading, yielding occurs when $\sigma_{m}=\hat{p}_{Y}^{-}$, with

$$
\hat{\mathrm{p}}_{Y}^{-}=\frac{\sigma_{x}^{T}}{3} \sqrt{\frac{3}{\hat{\mathrm{m}}^{2}\left(3 k^{2}-2 k+3\right)}\left(\frac{4 t_{1}+6 t_{2}}{5}\right)} \ln (f),
$$

the expressions for $\hat{m}, t_{1}$, and $t_{2}$ in terms of the orthotropy coefficients and $k$ being given by Eqs. (6), (9a-9b) and (A.1), respectively. Furthermore, according to the Stewart and Cazacu (2011) criterion, the yield function of the porous solid is no longer invariant with respect to the transformation $\left(\sigma_{\mathrm{m}}, \boldsymbol{\sigma}^{\prime}\right) \rightarrow\left(\sigma_{\mathrm{m}},-\boldsymbol{\sigma}^{\prime}\right)$.

\section{Plastic deformation in titanium: characterization tests and model identification}

\subsection{Experimental results in uniaxial compression and uniaxial tension}

The material used in this work is a high-purity (99.9\%) titanium, and it was purchased from Tico Titanium, Inc. The
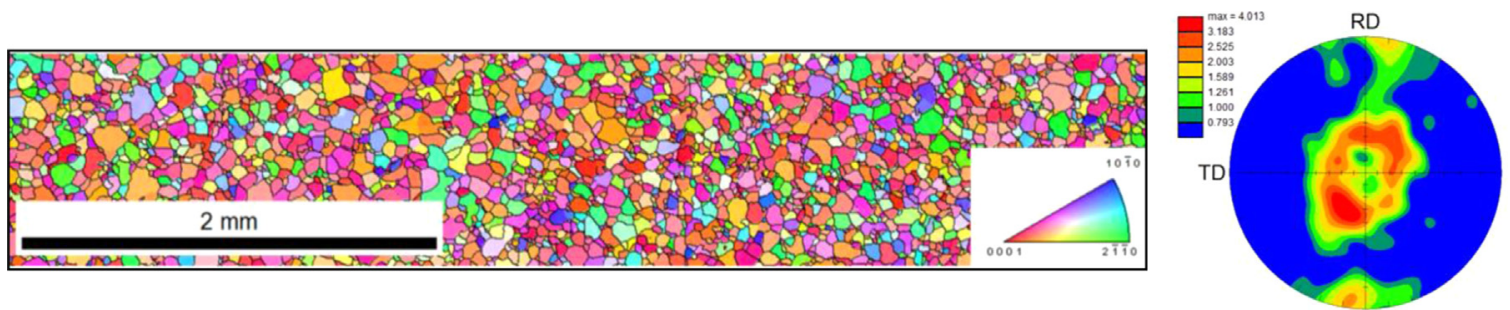

Fig. 1. Orientation map and $\{0001\}$ pole figure showing the initial texture of the hcp-titanium plate material, measured using Electron Back Scattered Diffraction (EBSD). The rolling directions (RD) and transverse direction (TD) are in the plane of the plate. 


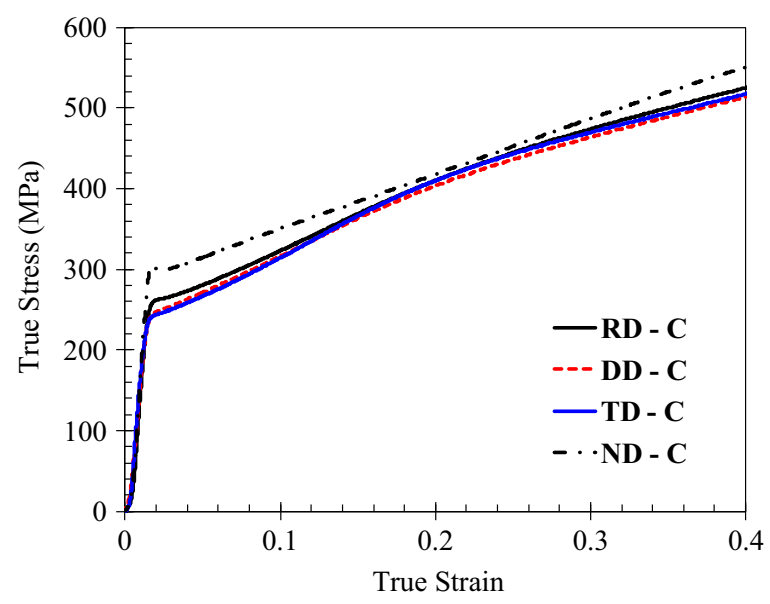

Fig. 2. Experimental quasi-static uniaxial compression stress-strain curves along several in-plane orientations $\theta$ measured with respect to the rolling direction (RD) (i.e. $\theta=0^{\circ}(\mathrm{RD}), 45^{\circ}(\mathrm{DD})$ and $90^{\circ}(\mathrm{TD})$ and in the normal direction (ND) of the hcp-titanium plate.

material was supplied in the form of a rolled plate with dimensions $610 \times 610 \times 16 \mathrm{~mm}^{3}$. Optical microscopy showed that the material has equiaxed grains with an average grain size of about $40 \mu \mathrm{m}$. The initial texture of the as received material was measured using Electron Back Scattered Diffraction (EBSD). Analysis of the $\{0001\}$ pole figure shows that the material has a basal texture, with the majority of the grains having their $c$-axis at $30^{\circ}$ to the normal to the plane of the plate (see Fig. 1). In order to quantify the influence of the loading direction, and thereby texture on the mechanical response at roomtemperature quasi-static tests (nominal strain rate of $0.01 \mathrm{~s}^{-1}$ ) in uniaxial compression were conducted on cylindrical specimens $7.62 \mathrm{~mm}$ in diameter by $7.62 \mathrm{~mm}$ long that were machined such that the axes of the cylinders were along the rolling direction (RD) and two other in-plane directions, at $45^{\circ}(\mathrm{DD})$ and $90^{\circ}$ (TD) with respect to RD, respectively. In addition, tests were conducted on specimens with the axis along the normal direction (ND) of the plate. It is to be noted that the uniaxial compression stress-strain curves along the in-plane directions are very close, but there is a marked difference between the yield stress in the normal direction and the average in-plane yield stresses (see Fig. 2). In-plane quasi-static tension tests (nominal strain rate of $0.01 \mathrm{~s}^{-1}$ ) were also conducted on flat dog-bone specimens, the rectangular cross section within the gauge length being $3.2 \mathrm{~mm}$ by $1.6 \mathrm{~mm}$. Digital image correlation (DIC) techniques were used to determine the strain fields (axial and width strains) in the gage zone. To assess the repeatability and consistency of the test results, five tests were performed for each orientation. The average uniaxial tensile true stress-true strain curves in $\mathrm{RD}, 45^{\circ}$, and TD are shown in Fig. 3. It is worth noting that this titanium material displays a strong and evolving tension-compression asymmetry, irrespective of the in-plane loading orientation, the strength in uniaxial compression being higher than the strength in unixial tension (see Fig. 4 showing the tension-compression asymmetry of the material for loadings along TD). As for most high-purity Ti materials, the difference in hardening between uniaxial tension and uniaxial compression may be due to deformation twinning and its polarity (e.g. Salem et al., 2003; Nixon et al., 2010a, Benhenni et al., 2013). Given that the uniaxial tension and compression stress-strain curves along different in-plane directions are close (see Figs. 2-3), one may

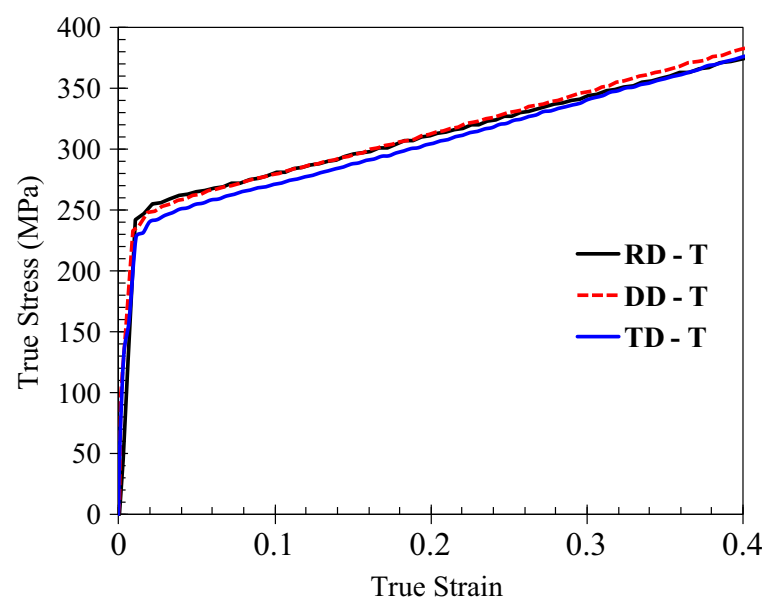

Fig. 3. Experimental quasi-static uniaxial tension stress-strain curves along several in-plane orientations $\theta$ measured with respect to the rolling direction (RD) (i.e. $\theta=0^{\circ}(\mathrm{RD}), 45^{\circ}(\mathrm{DD})$, and $90^{\circ}$ (TD) of the hcp-titanium plate. 


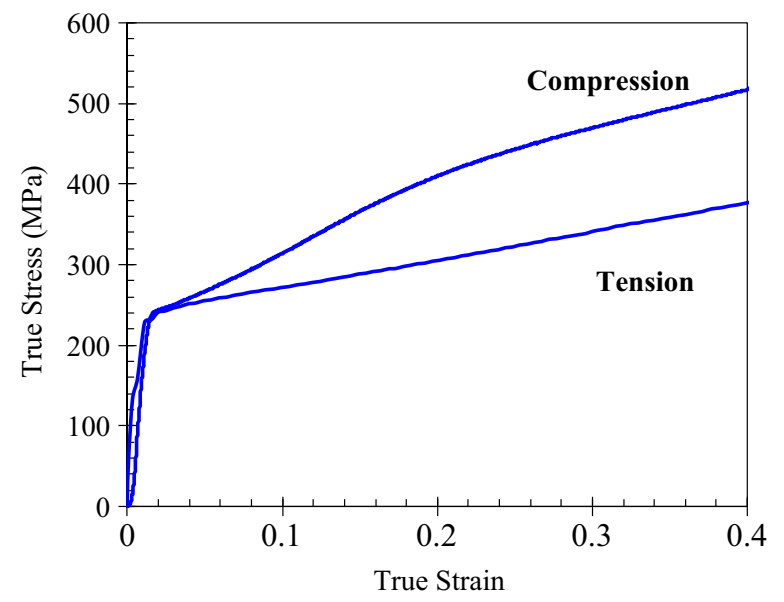

Fig. 4. Experimental evidence of the difference in response between uniaxial tension and compression along the in-plane transverse direction (TD) of the hcp-titanium plate material. Note that the material is harder in compression than in tension.

conclude that the material's in-plane anisotropy can be neglected. If this would be the case, the Lankford coefficients or r-values should be the same irrespective of the in-plane orientation $\theta$.

Indeed, the plastic strain ratio, $r(\theta)$, is defined as the ratio of the width to thickness plastic strain increments measured in a uniaxial tension test along an in-plane direction at an angle $\theta$ with respect to RD, i.e., $r_{\theta}=\dot{\epsilon}_{\text {width }} / \dot{\epsilon}_{\text {thickness. }}$. Generally, the $r$ values are obtained from measurements of the axial and width strains, the thickness strain being determined assuming plastic incompressibility (for example, see Barlat and Richmond, 1987). For each specimen orientation, $r$-values were determined for several levels of plastic strains and are listed in Table 1.

It is worth noting that there is a clear anisotropy in $r$-values, irrespective of the level of strain, the largest $r$-value being along RD. Thus, the material cannot be considered transversely isotropic.

Note also that the $r$-values are very large. This indicates that the material displays resistance to thinning, and that from a formability standpoint it is very challenging. For comparison, for an isotropic material, $r(\theta)=1$, irrespective of the tensile loading direction $\theta$, for a typical aluminum alloy, e.g. AA 3103-O, the $r(\theta)$ range between 0.5 and 0.6 (see Barlat et al., 2004). In conclusion on the basis of the mechanical characterization tests conducted, it can be concluded that the material displays orthotropy and tension-compression asymmetry.

\subsection{Identification of the parameters of Stewart and Сazacu (2011) model}

All the parameters involved in the criterion $\varphi(\sigma, f)$ have a clear physical meaning, being related to the plastic properties of the material. Specifically, all the parameters are expressible in terms of the anisotropy coefficients $\mathrm{L}_{i j}$ and/or the strengthdifferential parameter $k$ (see Eqs. (3-10)). For the identification of these parameters, the experimental data obtained in uniaxial tension on flat specimens and in uniaxial compression tests were used (see Appendix B for details on the identification procedure).

The evolution of the anisotropy in $r$-values with accumulated plastic deformation (see Table 1 ) and the evolving difference in hardening rates between tension and compression loadings observed from the test results are indicative of texture evolution (e.g. see Fig. 4). To describe these effects, the anisotropy coefficients and $k$ are considered to evolve with accumulated plastic deformation. The numerical values of these parameters corresponding to four individual levels of equivalent plastic strains (up to 0.35 strain) are listed in Table 2, the values corresponding to any given level of plastic strain $\bar{\varepsilon}_{p}^{j} \leq \bar{\varepsilon} \leq \bar{\varepsilon}_{p}^{j+1}$ being obtained by linear interpolation, i.e.:

$$
\begin{aligned}
& \mathrm{L}_{i j}(\bar{\epsilon})=\alpha(\bar{\epsilon}) \mathrm{L}_{i j}\left(\bar{\epsilon}_{\mathrm{p}}^{\mathrm{j}}\right)+(1-\alpha(\bar{\epsilon})) \mathrm{L}_{i j}\left(\bar{\epsilon}_{\mathrm{p}}^{j+1}\right) \\
& k(\bar{\epsilon})=\alpha(\bar{\epsilon}) \mathrm{k}\left(\bar{\epsilon}_{p}^{j}\right)+(1-\alpha(\bar{\epsilon})) \mathrm{k}\left(\bar{\epsilon}_{p}^{j+1}\right)
\end{aligned}
$$

Table 1

Lankford coefficients ( $r$-values) for several in-plane orientations $\theta$, measured with respect to the rolling direction. For each orientation, the values corresponding to several levels of effective plastic strain are reported.

\begin{tabular}{lllllll}
\hline Orientation $\theta(\mathrm{deg})$ & $\bar{\varepsilon}^{p}=0$ & $\overline{\bar{\varepsilon}}^{p}=0.05$ & $\overline{\bar{\varepsilon}}^{p}=0.1$ & $\overline{\bar{\varepsilon}}^{p}=0.15$ & $\overline{\bar{\varepsilon}}^{p}=0.2$ & $\overline{\bar{\varepsilon}}^{p}=0.25=0.35$ \\
\hline $0^{\circ}$ & 2.87 & 2.68 & 2.52 & 2.38 & 2.26 & 2.15 \\
$45^{\circ}$ & 2.08 & 2.01 & 1.94 & 1.88 & 1.82 & 1.78 \\
$90^{\circ}$ & 1.57 & 1.56 & 1.55 & 1.53 & 1.52 & 1.74 \\
\hline
\end{tabular}


Table 2

Model parameters for the high-purity orthotropic hcp-titanium corresponding to different values of the effective plastic strain, $\bar{\varepsilon}^{p}$; for any strain level $\mathrm{L}_{11}$ is set to unity.

\begin{tabular}{|c|c|c|c|c|c|c|c|}
\hline $\bar{\varepsilon}^{p}$ & $\mathrm{~L}_{22}$ & $\mathrm{~L}_{33}$ & $\mathrm{~L}_{12}$ & $\mathrm{~L}_{13}$ & $\mathrm{~L}_{23}$ & $\mathrm{~L}_{44}$ & $\mathrm{k}$ \\
\hline 0.02 & 0.971 & 1.316 & 0.022 & 0.189 & 0.152 & 0.972 & -0.304 \\
\hline 0.05 & 0.989 & 1.243 & 0.089 & 0.193 & 0.173 & 0.909 & -0.313 \\
\hline 0.1 & 0.992 & 1.046 & 0.016 & 0.075 & 0.053 & 0.983 & -0.363 \\
\hline 0.15 & 0.996 & 0.915 & -0.015 & 0.021 & 0.000 & 1.016 & -0.419 \\
\hline 0.2 & 0.998 & 0.849 & -0.048 & -0.012 & -0.034 & 1.050 & -0.472 \\
\hline 0.25 & 0.998 & 0.815 & -0.089 & -0.041 & -0.068 & 1.092 & -0.518 \\
\hline 0.3 & 0.998 & 0.797 & -0.130 & -0.068 & -0.099 & 1.134 & -0.554 \\
\hline 0.35 & 1.000 & 0.772 & -0.178 & -0.097 & -0.135 & 1.183 & -0.635 \\
\hline
\end{tabular}

The interpolation parameter $\alpha$ involved in Eq. (16) is defined as

$$
\alpha=\frac{\bar{\epsilon}-\bar{\epsilon}_{p}^{j}}{\bar{\epsilon}_{p}^{j+1}-\bar{\epsilon}_{p}^{j}} .
$$

It is considered that the material's hardening is isotropic and it is governed by the equivalent plastic strain according to a power-law:

$$
Y\left(\bar{\epsilon}^{p}\right)=\beta\left(\epsilon_{0}+\bar{\epsilon}^{p}\right)^{n},
$$
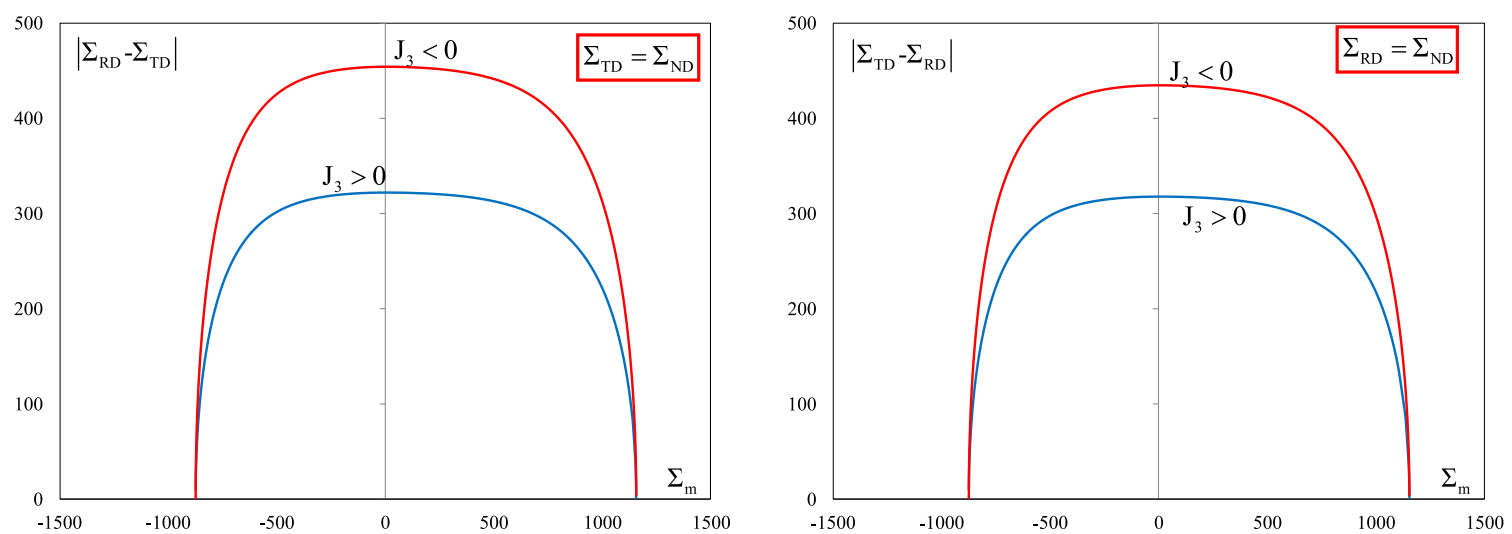

(a)

(b)

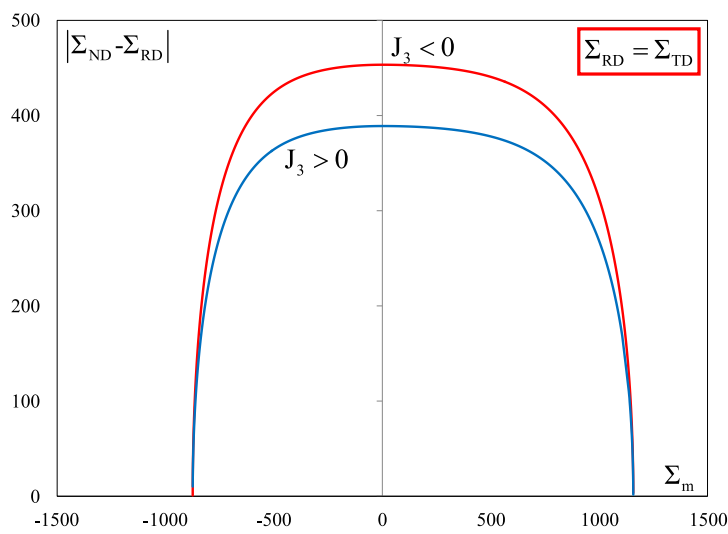

(c)

Fig. 5. Stewart and Cazacu (2011) surfaces for the porous orthotropic hcp-titanium plate corresponding to a porosity $f=0.01$ under axisymmetric loadings with axial stress along different orientations corresponding to either $\mathrm{J}_{3}>0$ or $\mathrm{J}_{3}<0$ (a) axisymmetric loading along RD-axis (J $>0$ corresponds to $\left.\Sigma_{\mathrm{RD}}>\Sigma_{\mathrm{ND}}=\Sigma_{\mathrm{TD}}\right)$ and $\left(\mathrm{J}_{3}<0\right.$ corresponds to $\Sigma_{\mathrm{RD}}<\Sigma_{\mathrm{ND}}=\Sigma_{\mathrm{TD}}$ ); (b) axisymmetric loading along TD-axis $\left(\mathrm{J}_{3}>0\right.$ corresponds to $\left.\Sigma_{\mathrm{TD}}>\Sigma_{\mathrm{RD}}=\Sigma_{\mathrm{ND}}\right)$ and (J3 $<0$ corresponds to $\Sigma_{\mathrm{TD}}<\Sigma_{\mathrm{RD}}=\Sigma_{\mathrm{ND}}$ ), and (c) axisymmetric loading along ND-axis $\left(\mathrm{J}_{3}>0\right.$, i.e. $\left.\Sigma_{\mathrm{ND}}>\Sigma_{\mathrm{RD}}=\Sigma_{\mathrm{TD}}\right)$ and $\left(\mathrm{J}_{3}<0\right.$, i.e. $\left.\Sigma_{\mathrm{ND}}<\Sigma_{\mathrm{RD}}=\Sigma_{\mathrm{TD}}\right)$, respectively. 
In Eq. (18), $\beta$ and $\varepsilon_{0}$ are material parameters, which can be estimated based on the experimental uniaxial tension axial stress vs. true strain curve in RD. The values of these parameters for the material studied are: $\beta=413 \mathrm{MPa}, \varepsilon_{0}=0.6445$ and $n=1$.

Note that Stewart and Cazacu (2011) criterion for porous solids (Eq. (3)) depends not only on all stress invariants, but also on the mixed invariants associated with orthotropy. As a consequence, even for the simplest axisymmetric loadings, the yield locus does not display the usual properties of existing criteria for porous solids, whether phenomenological or micromechanics-based. To illustrate the combined effects of the anisotropy and tension-compression asymmetry of the plastic flow on yielding of the porous Ti material studied, in Fig. 5 are shown the projections of the yield surface corresponding to an equivalent plastic strain $\bar{\epsilon}^{p}=0.25$ and a porosity $f=0.01$ for axisymmetric loadings with axial stress oriented either along the RD ( $x$-axis) (Fig. 5(a)), TD ( $y$-axis) (Fig. 5(b)) or normal direction (ND) ( $z$ - axis) (Fig. 5(c)), respectively. In each case, loadings corresponding to the axial stress being the minor principal stress (i.e. third-invariant, $\mathrm{J}_{3} \leq 0$ ) and loadings such that axial stress is the major principal stress (i.e. third-invariant, $\mathrm{J}_{3} \geq 0$ ) were considered. Note that irrespective of the orientation of the axial stress, it is predicted a strong effect of the third-invariant, $\mathrm{J}_{3}$, on yielding. The explanation for this strong influence of the ordering of the principal stresses is provided in the following.

Let first analyze the dilatational response of the material under axisymmetric loadings with axial stress along the RDaxis, for which $\mathrm{J}_{3}=2\left(\left(\Sigma_{\mathrm{RD}}-\Sigma_{\mathrm{TD}}\right)^{3} / 27\right.$ and $\Sigma_{\mathrm{m}}=\left(\Sigma_{\mathrm{RD}}+2 \Sigma_{\mathrm{TD}}\right) / 3$ (see Fig. 5(a)). Note that if the minor principal stress is along RD and $\Sigma_{\mathrm{TD}}=\Sigma_{\mathrm{ND}}$ (i.e. $\mathrm{J}_{3} \leq 0$ ) then the criterion writes:

$$
\varphi= \begin{cases}\left(\frac{\sigma_{x}^{T}}{\sigma_{x}^{C}}\right)^{2}\left(\frac{\Sigma_{\mathrm{RD}}-\Sigma_{\mathrm{TD}}}{\sigma_{x}^{T}}\right)^{2}+2 f \cosh \left(\frac{\Sigma_{\mathrm{RD}}+2 \Sigma_{\mathrm{TD}}}{3 \sigma_{x}^{T}} \sqrt{\frac{15 \hat{m}^{2}\left(3 k^{2}-2 k+3\right)}{4 t_{1}+6 t_{2}}}\right)-\left(1+f^{2}\right), & \text { if } \Sigma_{\mathrm{m}}<0, \\ \left(\frac{\sigma_{x}^{T}}{\sigma_{x}^{C}}\right)^{2}\left(\frac{\Sigma_{\mathrm{RD}}-\Sigma_{\mathrm{TD}}}{\sigma_{x}^{T}}\right)^{2}+2 f \cosh \left(\frac{\Sigma_{\mathrm{RD}}+2 \Sigma_{\mathrm{TD}}}{3 \sigma_{x}^{T}} \sqrt{\frac{15 \hat{m}^{2}\left(3 k^{2}+2 k+3\right)}{4 t_{1}+6 t_{2}}}\right)-\left(1+f^{2}\right), & \text { if } \Sigma_{\mathrm{m}} \geq 0 .\end{cases}
$$

However, if the minor principal value of the applied stress is along TD and $\Sigma_{\mathrm{TD}}=\Sigma_{\mathrm{ND}} \leq \Sigma_{\mathrm{RD}}$ (i.e. $\mathrm{J}_{3} \geq 0$ ) then:

$$
\varphi=\left\{\begin{array}{l}
\left(\frac{\Sigma_{\mathrm{RD}}-\Sigma_{\mathrm{TD}}}{\sigma_{x}^{T}}\right)^{2}+2 f \cosh \left(\frac{\Sigma_{\mathrm{RD}}+2 \Sigma_{\mathrm{TD}}}{3 \sigma_{x}^{T}} \sqrt{\frac{15 \hat{m}^{2}\left(3 k^{2}-2 k+3\right)}{4 t_{1}+6 t_{2}}}\right)-\left(1+f^{2}\right), \quad \text { if } \Sigma_{\mathrm{m}}<0, \\
\left(\frac{\Sigma_{\mathrm{RD}}-\Sigma_{\mathrm{TD}}}{\sigma_{x}^{T}}\right)^{2}+2 f \cosh \left(\frac{\Sigma_{\mathrm{RD}}+2 \Sigma_{\mathrm{TD}}}{3 \sigma_{x}^{T}} \sqrt{\frac{15 \hat{m}^{2}\left(3 k^{2}+2 k+3\right)}{4 t_{1}+6 t_{2}}}\right)-\left(1+f^{2}\right), \quad \text { if } \Sigma_{\mathrm{m}} \geq 0,
\end{array}\right.
$$

with $\sigma_{\mathrm{x}}^{\mathrm{c}}$ the matrix uniaxial compressive flow stress in the $\mathrm{x}$ direction. Note that the ratio between the predicted yield values corresponding to the same triaxiality is maximum for purely deviatoric loadings (triaxiality zero, $\Sigma_{\mathrm{m}}=0$ ) when this ratio is equal to $\sigma_{x}^{T} / \sigma_{x}^{C}$. Since the material studied is harder in uniaxial compression than in uniaxial tension $\left(\sigma_{x}^{T} / \sigma_{x}^{C}<1\right)$, from Eq. (19) it follows that the overall response is softer under axisymmetric loadings corresponding to $\mathrm{J}_{3}>0$ than under axisymmetric loadings corresponding to $\mathrm{J}_{3}<0$, and as a consequence the strains at failure will also be markedly different (see also, Fig. 5 (a)).

On the other hand, due to the material's plastic anisotropy under axisymmetric loadings for which the axial stress along the TD-axis $\left(\mathrm{J}_{3}=2\left(\Sigma_{\mathrm{TD}}-\Sigma_{\mathrm{RD}}\right)^{3} / 27\right.$ and $\left.\Sigma_{\mathrm{m}}=\left(2 \Sigma_{\mathrm{RD}}+\Sigma_{\mathrm{TD}}\right) / 3\right)$, if the minor axial stress is along RD, (i.e. $\mathrm{J}_{3} \geq 0$ )

$$
\varphi=\left\{\begin{array}{l}
\left(\frac{\sigma_{x}^{T}}{\sigma_{y}^{C}}\right)^{2}\left(\frac{\Sigma_{\mathrm{TD}}-\Sigma_{\mathrm{RD}}}{\sigma_{x}^{T}}\right)^{2}+2 f \cosh \left(\frac{\Sigma_{m}}{\sigma_{x}^{T}} \sqrt{\frac{15 \hat{m}^{2}\left(3 k^{2}-2 k+3\right)}{4 t_{1}+6 t_{2}}}\right)-\left(1+f^{2}\right), \quad \text { if } \Sigma_{\mathrm{m}}<0, \\
\left(\frac{\sigma_{x}^{T}}{\sigma_{y}^{C}}\right)^{2}\left(\frac{\Sigma_{\mathrm{TD}}-\Sigma_{\mathrm{RD}}}{\sigma_{x}^{T}}\right)^{2}+2 f \cosh \left(\frac{\Sigma_{m}}{\sigma_{x}^{T}} \sqrt{\frac{15 \hat{m}^{2}\left(3 k^{2}+2 k+3\right)}{4 t_{1}+6 t_{2}}}\right)-\left(1+f^{2}\right), \quad \text { if } \Sigma_{\mathrm{m}} \geq 0 .
\end{array}\right.
$$

(compare with Eq. (19a)), while for loadings for which the minor axial stress is along TD, but $\Sigma_{\mathrm{ND}}=\Sigma_{\mathrm{RD}}$ the criterion writes: 


$$
\varphi=\left\{\begin{array}{l}
\left(\frac{\sigma_{x}^{T}}{\sigma_{y}^{T}}\right)^{2}\left(\frac{\Sigma_{\mathrm{TD}}-\Sigma_{\mathrm{ND}}}{\sigma_{x}^{T}}\right)^{2}+2 f \cos h\left(\frac{\Sigma_{m}}{\sigma_{x}^{T}} \sqrt{\frac{15 \hat{m}^{2}\left(3 k^{2}-2 k+3\right)}{4 t_{1}+6 t_{2}}}\right)-\left(1+f^{2}\right), \quad \text { if } \Sigma_{\mathrm{m}}<0, \\
\left(\frac{\sigma_{x}^{T}}{\sigma_{y}^{T}}\right)^{2}\left(\frac{\Sigma_{\mathrm{TD}}-\Sigma_{\mathrm{ND}}}{\sigma_{T}}\right)^{2}+2 f \cos h\left(\frac{\Sigma_{m}}{\sigma_{x}^{T}} \sqrt{\frac{15 \hat{m}^{2}\left(3 k^{2}+2 k+3\right)}{4 t_{1}+6 t_{2}}}\right)-\left(1+f^{2}\right), \quad \text { if } \Sigma_{\mathrm{m}} \geq 0,
\end{array}\right.
$$

(compare Eq. (20b) with Eq. (19b)). In the above equations, $\sigma_{y}^{c}$ and $\sigma_{y}^{\mathrm{T}}$ denote the matrix uniaxial compressive and tensile yield stresses along the TD ( $\mathbf{y}$ axis), respectively.

Note that for axisymmetric loadings such that $\Sigma_{\mathrm{ND}}=\Sigma_{\mathrm{RD}}$, the model predicts that the maximum difference between the yield curves at $\mathrm{J}_{3}>0$ and $\mathrm{J}_{3}<0$ is exactly the ratio between the matrix flow stresses in tension and compression along the TD ( $y$-direction), i.e. $\sigma_{y}^{T} / \sigma_{y}^{C}$. (see also Fig. 5(b)). Similarly, it can be shown that for axisymmetric loadings such as $\Sigma_{\mathrm{ND}}=\Sigma_{\mathrm{TD}}$, the maximum split between the yield curves at $\mathrm{J}_{3}>0$ and $\mathrm{J}_{3}<0$ is exactly the ratio between the matrix ND flow stresses in tension and compression, i.e. $\sigma_{z}^{T} / \sigma_{z}^{C}$.

In summary, the sensitivity to the sign of $\mathrm{J}_{3}$ is due to the tension-compression asymmetry ratio in the direction of the applied axial stress. Given that for the material studied $\sigma_{x}^{T} / \sigma_{x}^{C}<1, \sigma_{y}^{T} / \sigma_{y}^{C}<1$ and $\sigma_{z}^{T} / \sigma_{z}^{C}<1$, the same general trends are observed for axisymmetric loadings with the axial stress along the RD axis, TD-axis and ND-axis respectively (compare Fig. 5 (a)-(c)). Namely, for axisymmetric loadings at $\mathrm{J}_{3}>0$ the response is softer than in the case of axisymmetric loadings at $\mathrm{J}_{3}<0$. However, the influence of the anisotropy of the material is clearly observed, the sensitivity to $\mathrm{J}_{3}$ being less pronounced for loadings with the axial stress along ND (see Fig. 5(a)-(c)). Furthermore, the yield surface lacks symmetry with respect to the deviatoric axis $\left(\Sigma_{\mathrm{m}}=0\right)$. In particular, the material's yield stress for purely tensile hydrostatic loadings is different than that under purely hydrostatic compressive loadings (see also Eq. (16)).

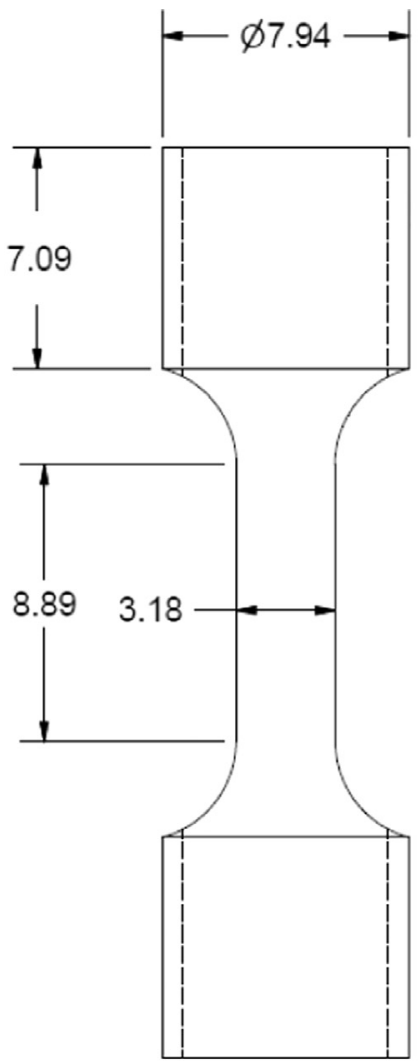

(a)

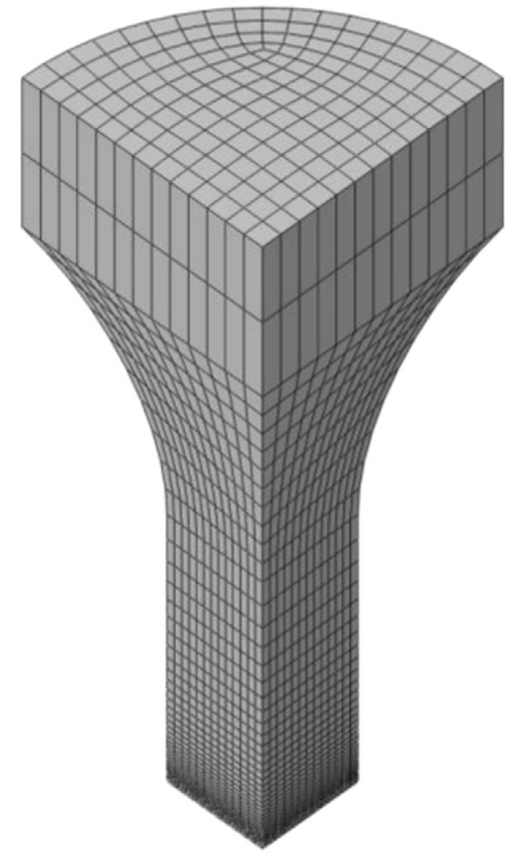

(b)

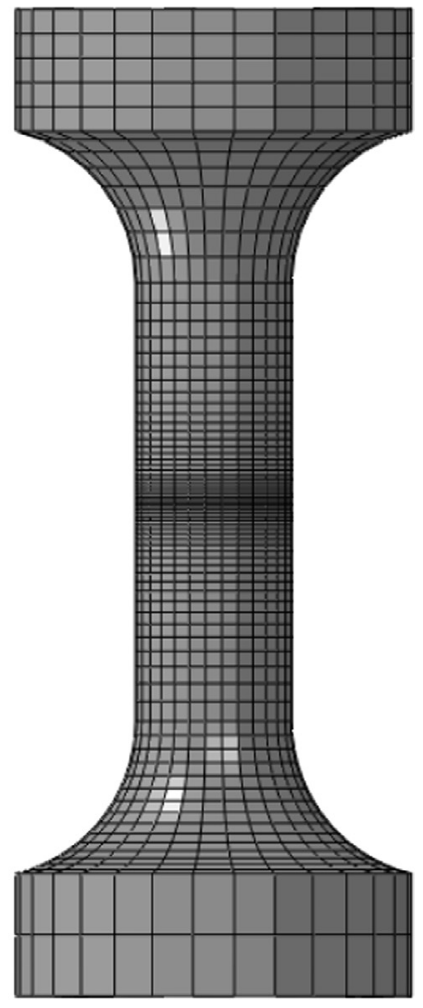

(c)

Fig. 6. (a) Geometries of the axisymmetric smooth tensile specimen); (b) F.E. meshes used for the specimens with axes along the directions of orthotropy: rolling direction (RD) and transverse direction (TD); (c) F.E. meshes used for the off-axis specimens i.e. the $15^{\circ}, 45^{\circ}$ and at $75^{\circ}$ to the rolling direction (RD). Dimensions are in mm. 


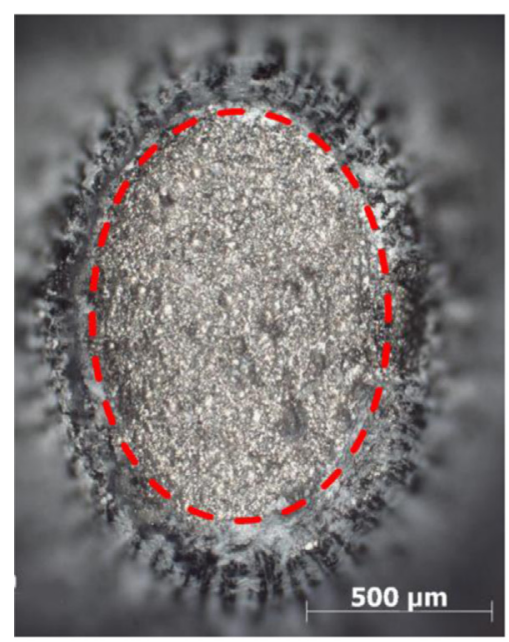

(a) $\mathrm{RD}$

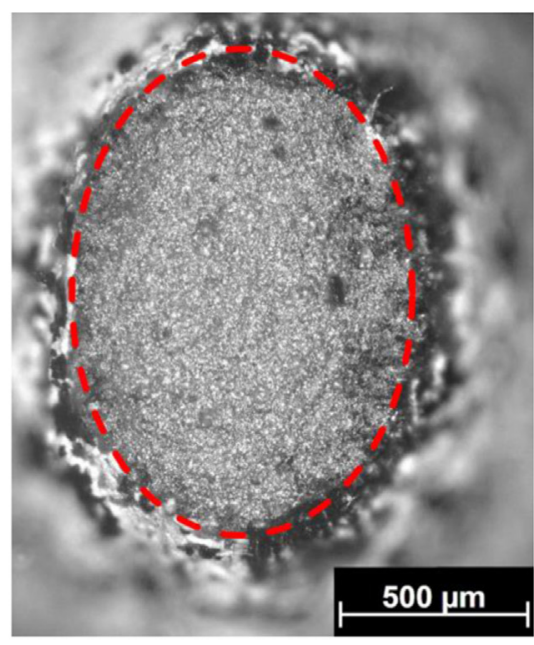

(b) $15^{\circ}$

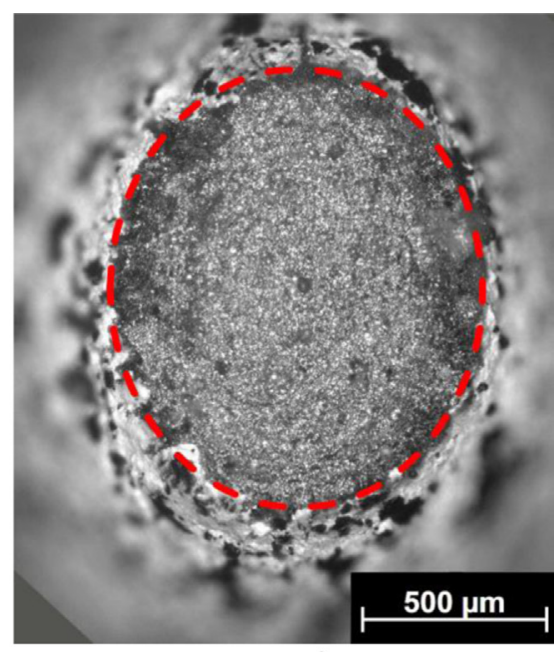

(c) $45^{\circ}$

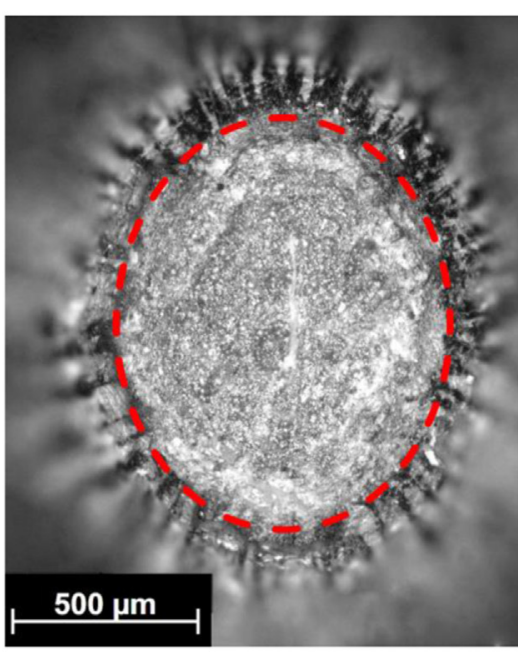

(d) $75^{\circ}$

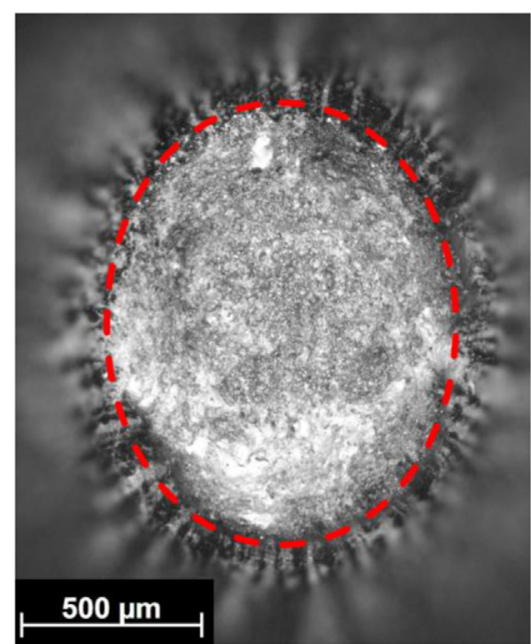

(e) $90^{\circ}$ (TD)

Fig. 7. Comparison between experimental and F.E. predictions according to the Stewart and Cazacu (2011) porous model of the final cross-sections of axisymmetric tensile specimens after uniaxial tension along different in-plane orientations: (a) RD; (b) $15^{\circ}$ to RD; (c) $45^{\circ}$ to RD; (d) $75^{\circ}$ to RD; $90^{\circ}$ to RD, respectively. The F.E. predictions are represented as dashed lines.

\section{X-ray micro-tomography measurements on specimens with circular cross-section and comparison between model and data}

Next, the capabilities of the model to predict plastic deformation and damage in the Ti material for loadings that were not used for identification are demonstrated. F.E. simulations of the uniaxial tension response of specimens of circular crosssection will be done and the F.E. predictions will be compared with the measured final cross-sections and X-ray computed micro-tomography (XCMT) measurements of porosity that we performed for both smooth and notched specimens. The presentation of those experimental tests is given in Section 4.1. All the simulations were conducted with the same numerical values for the parameters involved in the model. Recall that the model parameters are those associated to the plastic properties of the material, namely the anisotropy coefficients $\mathrm{L}_{\mathrm{ij}}$, strength differential parameter $k$ (see Table 2), and the parameters associated to the isotropic hardening of the matrix material (Eq. (18)). The values of these parameters were determined from tensile tests on specimens with rectangular cross-section and compression tests (see Section 3). The average initial value of the void volume fraction of the specimens was estimated to be: $f_{0}=0.0001$. The numerical values of the parameters involved in the void nucleation law (Eq.(13)) are: $f_{\mathrm{N}}=0.001, S_{\mathrm{N}}=0.4, \varepsilon_{\mathrm{N}}=0.9, f_{\mathrm{P}}=0.001, S_{\mathrm{P}}=250 \mathrm{MPa}$, $\sigma_{\mathrm{P}}=800 \mathrm{MPa}$. The elastic parameters values are: $E=110 \mathrm{GPa}, \nu=0.3$, where $\mathrm{E}$ is the Young modulus and $\nu$ is the Poisson coefficient. All the simulations were carried out using a user material subroutine (UMAT) that was developed for the constitutive model presented in Section 2 and implemented in the commercial implicit F.E. solver ABAQUS Standard (ABAQUS, 2009). A fully implicit integration algorithm was used for solving the governing equations. 


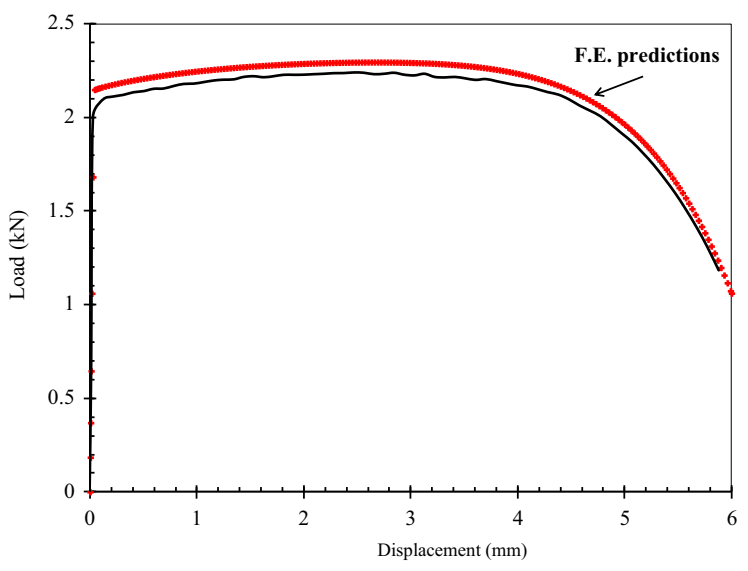

(a) $\mathrm{RD}$ specimen

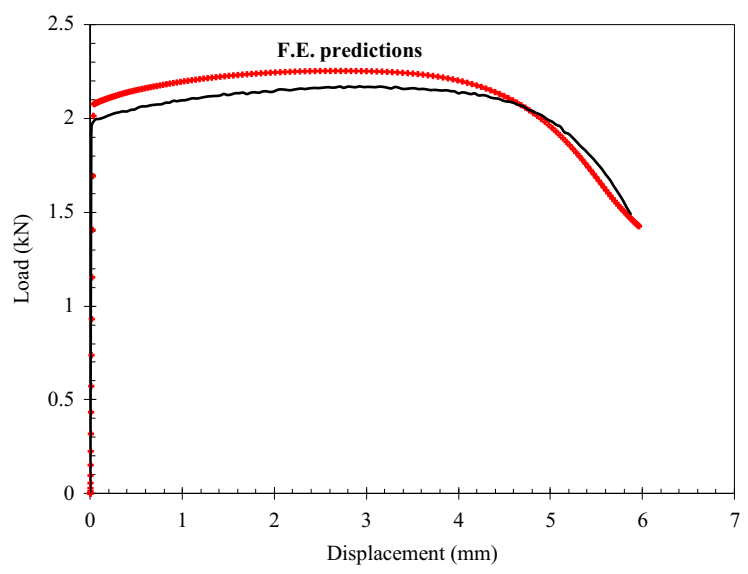

(b) $45^{\circ}$ specimen

Fig. 8. Comparison between experimental data and F.E. predictions of the load vs. axial displacement curves according to the Stewart and Cazacu (2011) porous model for uniaxial tension of smooth axisymmetric specimens (a) RD specimen, and (b) $45^{\circ}$ specimen.

\subsection{Experimental test results in uniaxial tension of axisymmetric smooth specimens and F.E. model predictions}

To further the understanding of plasticity-damage couplings of the material, we performed additional uniaxial tension tests on smooth specimens of circular cross-section (radius of $3.18 \mathrm{~mm}$ ) with axis along RD, TD, and at $15^{\circ}, 45^{\circ}$ and at $75^{\circ}$ to the rolling direction. The specimen geometry and the F.E. meshes used in the simulations are shown in Fig. 6 . Note that for the specimens oriented along the axes of orthotropy of the material (i.e. either RD or TD), only one-eighth of the specimen needs to be analyzed. The mesh used consisted of 8109 hexahedral elements (ABAQUS C3D8) (see Fig. 6(b)). However, for off-axes specimens $\left(15^{\circ}, 45^{\circ}\right.$ and at $\left.75^{\circ}\right)$ symmetric boundary conditions cannot be applied, and the entire specimens were meshed. While the F.E. mesh used in these cases consists of 16,355 hexahedral elements (see Fig. 6(c)), the size of the elements in the middle of the specimen (i.e. the zone of interest) is almost the same as for the RD and TD specimens.

To assess the predictive capabilities of the model, we first compare the predicted final cross-section with the one obtained experimentally in each test. In Fig. 7 are shown the photographs of the final cross-sections of the respective specimens on which are superposed the F.E. predictions obtained with the model (dashed lines). It is worth noting that for all
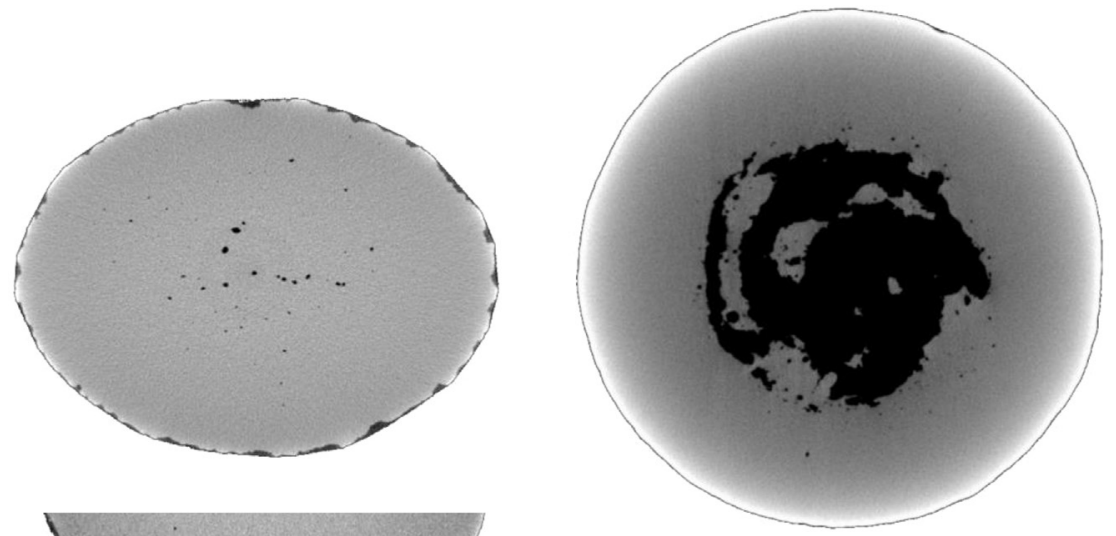

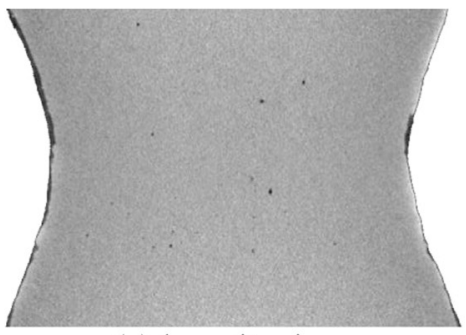

(a) hcp-titanium

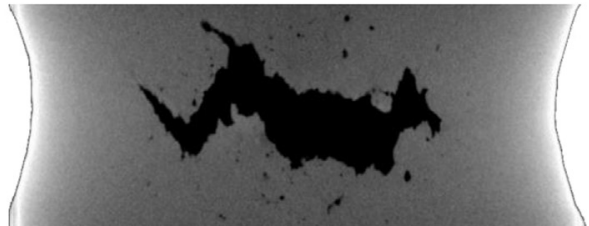

(b) Copper

Fig. 9. Post-test X-ray computed micro-tomography (XCMT) scans of smooth axisymmetric specimens of circular cross-sections that were subjected to uniaxial tension to large plastic deformations (close to failure): (a) hcp-titanium material studied; (b) isotropic copper material. 

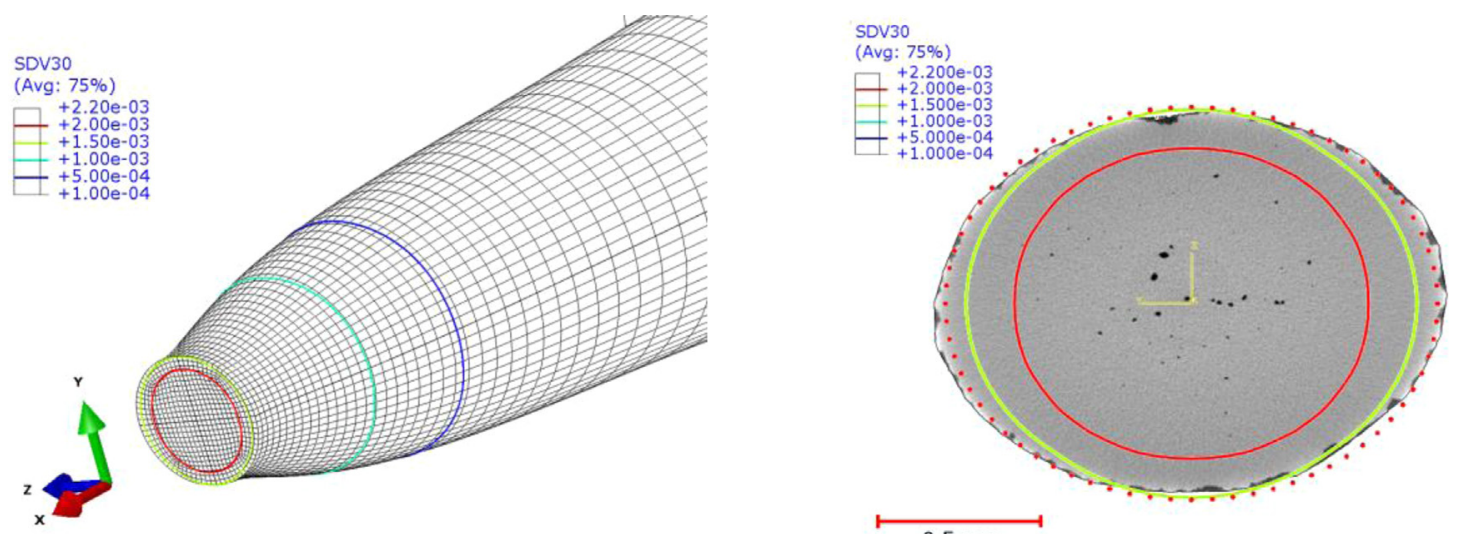

(a)
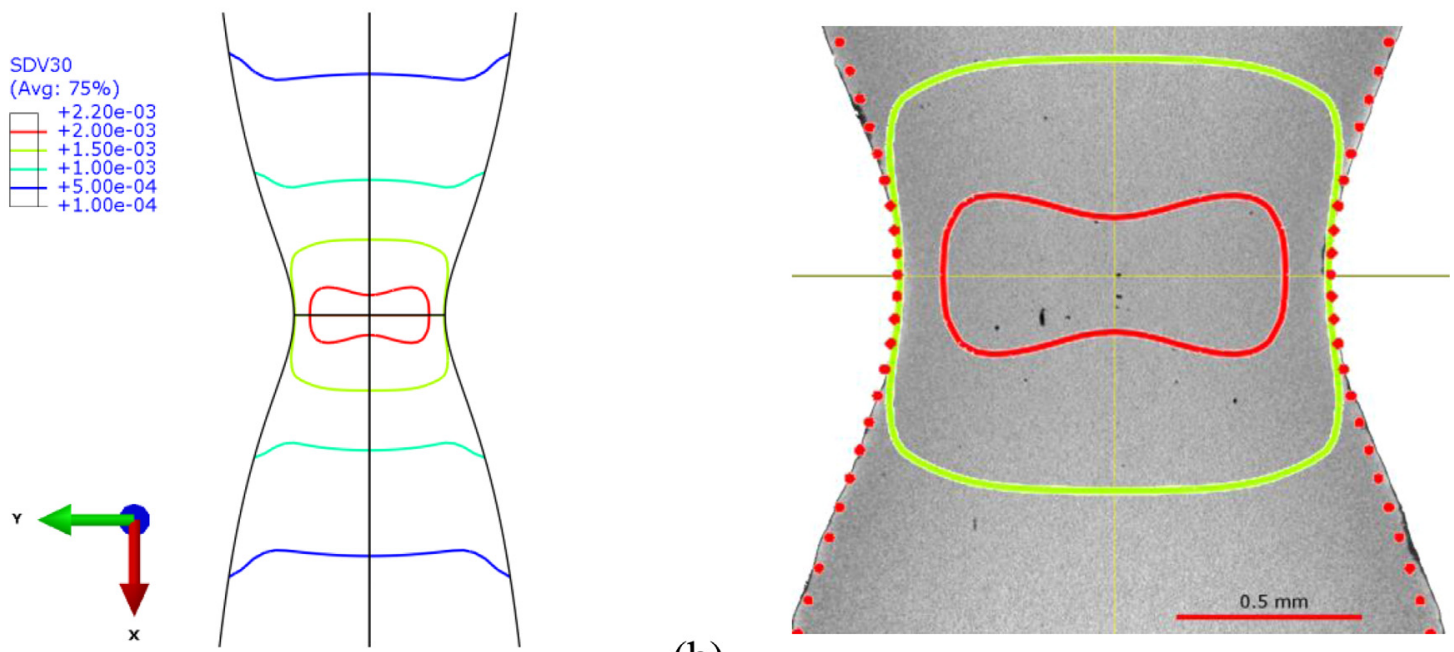

(b)
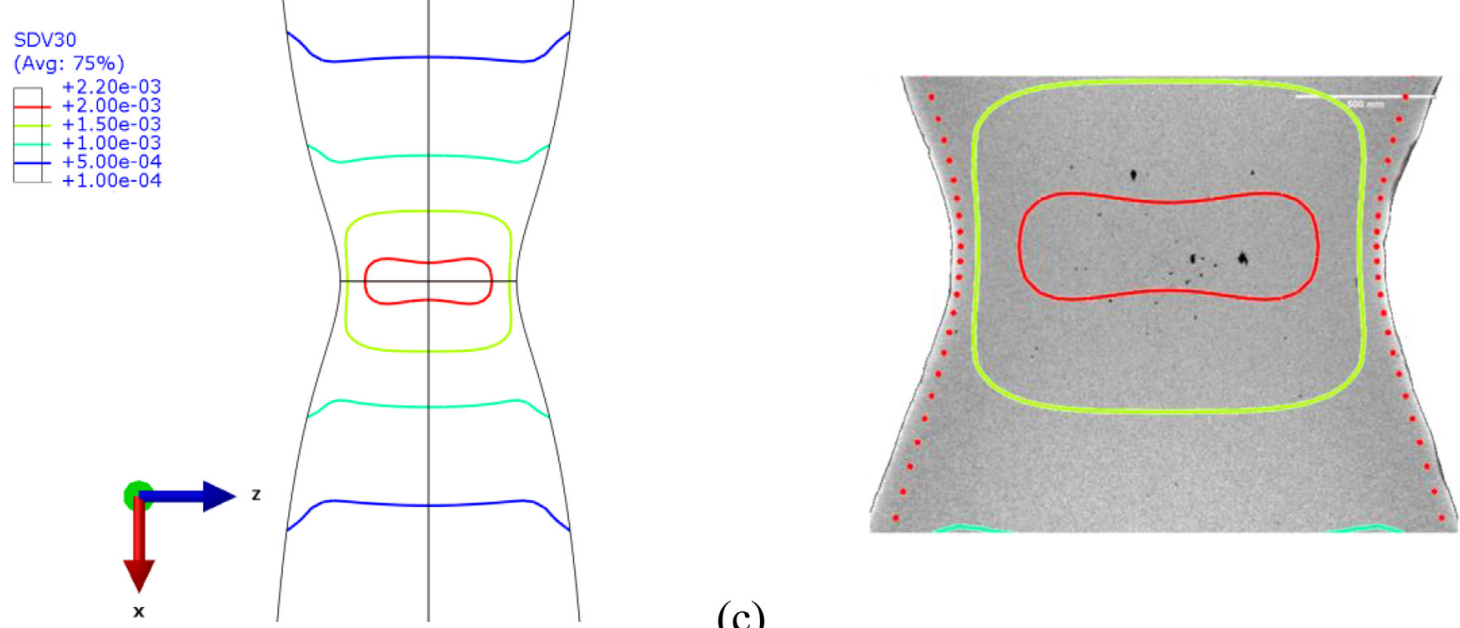

(c)

Fig. 10. Comparison between the F.E. cross-sections and isocontours of void volume fraction of a smooth axisymmetric specimen of hcp-titanium subjected to uniaxial tension along $\mathrm{x}$-direction (RD), according to the Stewart and Cazacu (2011) model and XCMT data for an axial notch displacement of 5.52 mm: (a) deformed specimen cross-section; (b) $(x-y)$ section of the deformed specimen; (c) (x-z) section of the deformed specimen. The axes $x, y, z$ are along the rolling (RD), transverse (TD), and normal (ND) directions. 
the loading orientations, the F.E. predictions are in good agreement with the experimental data. The Stewart and Cazacu (2011) porous model correctly captures the anisotropy in plastic deformation of the material, irrespective of the specimen orientation the initial circular cross-section becoming elliptical.

Let define the ellipticity $e$, as

$$
e=(a-b) / b \text {, }
$$

where $a$ and $b$, are the major and minor axes of the respective deformed cross-section. Stewart and Cazacu (2011) porous model predicts well the influence of the loading orientation on the shape of the final cross-section, the largest ellipticity being obtained for the RD specimen. Specifically, for the RD specimen, the F.E. predicted ellipticity is of $28.3 \%$ against $30.4 \%$ obtained experimentally. For the specimens oriented along other directions, the ellipticity predicted by the model is of $25 \%$ for the $15^{\circ}$ specimen (against $26.5 \%$ experimentally), $22 \%$ for the $45^{\circ}$ specimen (against $21.7 \%$ experimentally), $21 \%$ for the $75^{\circ}$ specimen (against 24\% experimentally) and 19.7\% (against $22.7 \%$ experimentally). Thus, it can be concluded that the model predictions are in quantitative agreement with the data.

It is also worth noting the Stewart and Cazacu (2011) model captures fairly well the experimental axial load vs. displacement curves for all specimens. As an example, in Fig. 8 are shown comparisons between the load-displacement curve obtained experimentally and theoretically for the RD specimen (Fig. 8(a)), and for the $45^{\circ}$ specimen, respectively (Fig. 8 (b)).

It is worth recalling that all the simulations were conducted with the same set of values for the model parameters which were identified based on compression data and tension data obtained on flat specimens (see also Appendix B). Thus, the simulation results presented in Fig. 7-8 are fully predictive of the plastic behavior of the material. Next, we will assess the capabilities of the model to predict porosity evolution in this hcp-titanium.

\subsection{XCMT measurements of damage for axisymmetric smooth $R D$ specimen}

With the availability of High-Resolution X-ray Computed Tomography (XRCT), in the past decade it has become possible the quantification of damage in the bulk of a given material. To have information on damage in the material under uniaxial tension, in the present work, images obtained by X-ray computed microtomography (using Xradia X-ray microscope, VersaXRM-500) were taken at the necking region of an axisymmetric smooth RD specimen. Tomography acquisition was carried out with a cubic voxel size of $1.2214^{3}=1.82 \mu \mathrm{m}^{3}$ and a range of view (ROV) of 2030 pixels. The total data set consisted of 1361 images with $2030 \times 2030$ pixels each, a total of about $5.6 \times 10^{9}$ voxels representing a volume of $2.48 \times 2.48 \times 1.66=10.2 \mathrm{~mm}^{3}$.The XCMT scan was done ex-situ, after the specimen was subjected to uniaxial tension to an axial displacement of $5.52 \mathrm{~mm}$. It is worth noting that the XCMT scan was taken very close to failure. In Fig. 9(a) are shown the reconstructed 2-D views in the (TD, TT) plane, and in the (RD, TD) plane of the specimen, respectively. For comparison purposes, in Fig. 9(b) are shown the respective views extracted from an XCMT scan of a copper specimen, which was taken at the same axial displacement. It is very important to note that for the same axial displacement, the copper material is very damaged while the titanium material shows very little damage. While a large hole/crack is seen in the middle of the copper specimen, almost no damage is observed in the hcp-Ti specimen. It means that for uniaxial tensile loading the rate of damage growth is slower in Ti than in $\mathrm{Cu}$. Thus, these XCMT observations confirm the conclusions of the preliminary theoretical study of Revil-Baudard and Cazacu (2013) on the influence of the tension-compression asymmetry on damage growth rate and damage distribution in a round tensile specimen which was done using the Cazacu and Stewart (2009) criterion which is the isotropic form of Stewart and Cazacu (2011) model (i.e. $L=I 4$, where I4 is the fourth-order unit tensor in Eq. (3)). Specifically, in that theoretical study it was shown that for materials for which the flow stress in uniaxial

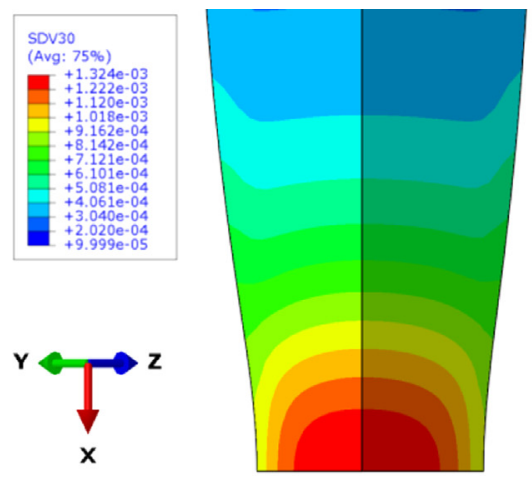

(a)

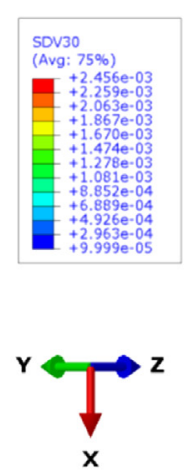

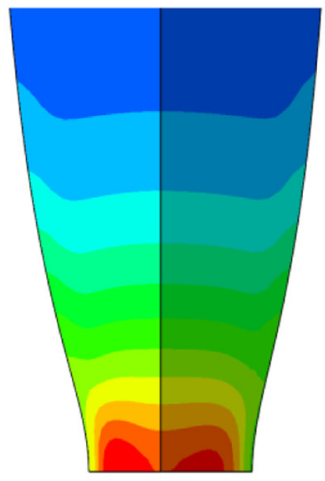

(b)

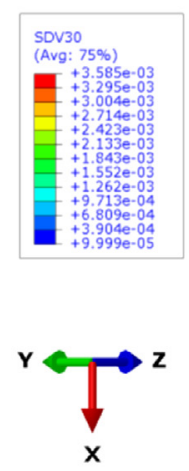

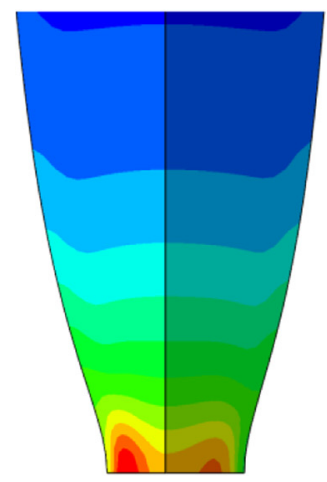

(c)

Fig. 11. F.E. predictions according to the Stewart and Cazacu (2011) model of the isocontours for the void volume fraction of an axisymmetric smooth specimen of hcp-titanium subjected to uniaxial tension along the x-direction (rolling direction RD) for other axial displacements: (a) 5 mm (b) 5.6 mm (c) $5.85 \mathrm{~mm}$. Note that for large axial displacement, the location of the zone of maximum void volume fraction shifts from the center. The axes $x, y, z$ are along the rolling (RD), transverse (TD), and normal (ND) directions. 
compression is larger than the flow stress in uniaxial tension damage is delayed as compared to materials for which the matrix plastic behavior is governed by the von Mises criterion. The observed drastic difference in damage evolution between hcp and titanium, which is harder in compression than in tension (see Fig. 4), and the copper material, which does not display tension-compression asymmetry validates the main conclusion of Revil-Baudard and Cazacu (2013) study, namely that the rate of void growth being much lower for Ti than for copper. In summary, Fig. 9 clearly shows that there is a very strong coupling between the specificities of the plastic deformation and porosity/damage evolution.

\subsection{Comparison between model prediction and XCMT measurements for an axisymmetric smooth RD specimen}

In order to assess the predictive capabilities of the Stewart and Cazacu (2011) porous model in terms of damage evolution in HCP-Ti, the XCMT data were compared with the F.E. isocontours of the void volume fraction corresponding to the same axial displacement (see Fig. 10). Specifically, the F.E. predictions are superposed on the different views obtained by XCMT, namely on the experimental cross-section, the experimental (RD, TD) section, and the experimental (RD, ND). To extract the experimental void volume fraction, a clustering technique was applied in order to identify 3-D groups of connected voxels, and thus to identify individually each pore within the matrix. A total of 2385 pores (independent cavities) has been detected in the total volume of the specimen, which resulted into an average porosity over the specimen volume of $0.052 \%$. It is worth noting that if only the pores of volume larger than 50 voxels are considered (i.e. number of 1857 independent cavities), the average void volume fraction in the total volume of the specimen is of $0.051 \%$.

Irrespective of the threshold value prescribed for pore size, the maximum average porosity corresponds to the root of the neck. The average porosity in the minimal cross-section (root of the neck) is of $0.25 \%$. It is worth noting that the Stewart and Cazacu (2011) model also predicts that damage is diffuse, in the minimal cross-section the maximum void volume fraction predicted being

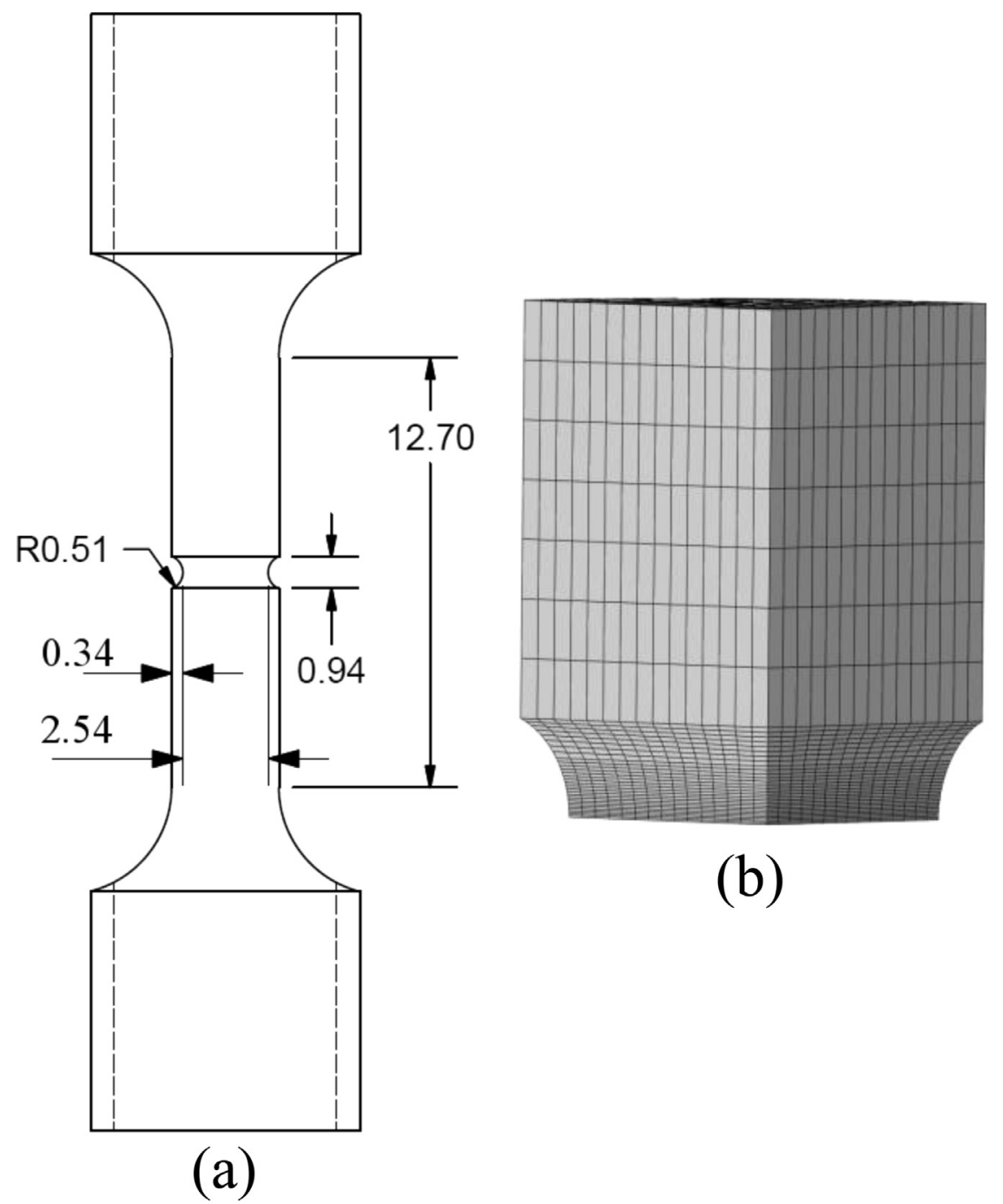

Fig. 12. Geometry and F.E. mesh for the notched specimen used for unixial tension along the rolling direction (RD). Dimensions are in mm. 


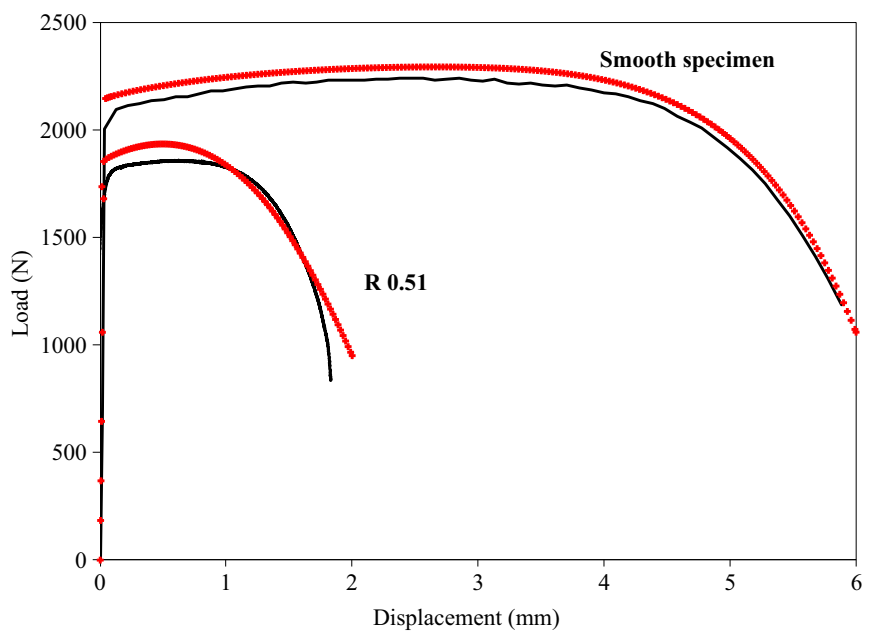

Fig. 13. Effect of the notch geometry on the load vs. displacement response for hcp-titanium according to the Stewart and Cazacu (2011) porous model and experimental data in uniaxial tension along the rolling direction.

of $0.22 \%$ (see Fig. 10(a)). Likewise, for the other views (Fig. 10(b) and (c), respectively), most of the voids observed by XCMT are inside the region of maximum void volume fraction predicted by the model. Furthermore, in Fig. 10, the F.E. predictions of the geometry of the specimen for the respective axial displacement (red points) are superposed on the different experimental cut views. It is to be noted that the Stewart and Cazacu (2011) model correctly predicts the section geometry in all the planes.

While XCMT data were obtained for an axial displacement of $5.52 \mathrm{~mm}$, it is interesting to analyze what would be the damage distribution corresponding to other axial displacements. For this purpose, in Fig. 11 are shown the F.E. predictions of the isocontours of the void volume fraction predicted for the same RD smooth specimen corresponding to a lower axial displacement (Fig. 11(a)) and to higher axial displacements of $5.6 \mathrm{~mm}$ (Fig. 11(b)), and $5.85 \mathrm{~mm}$ (Fig. 11(c)), respectively. It is

\section{Cross-section (TD,ND) cut view}

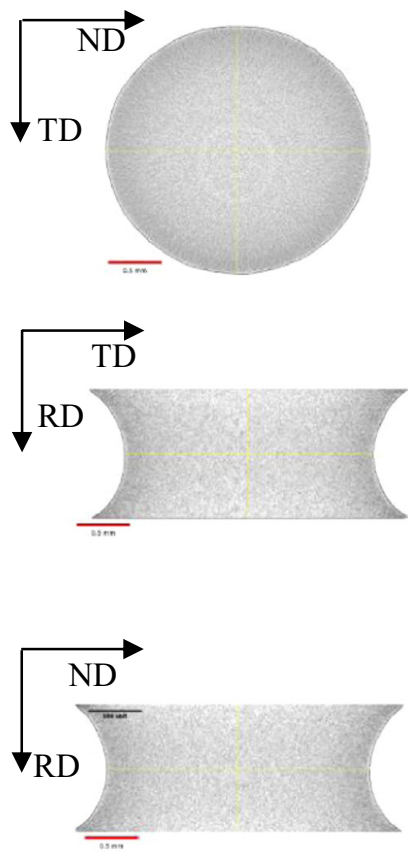

(a)

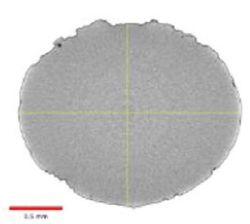

$(\mathrm{RD}, \mathrm{TD})$

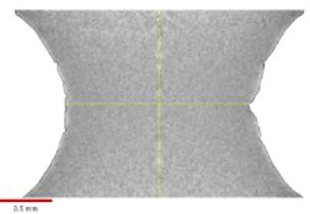

$(\mathrm{RD}, \mathrm{ND})$

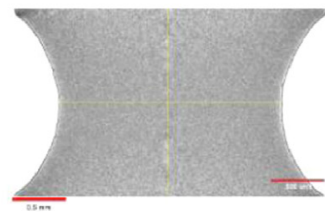

(b)

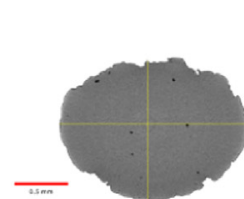

cut view

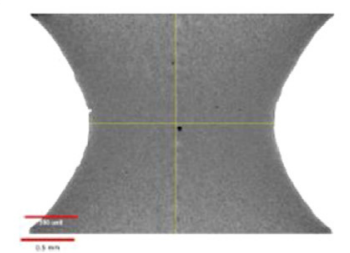

(c)
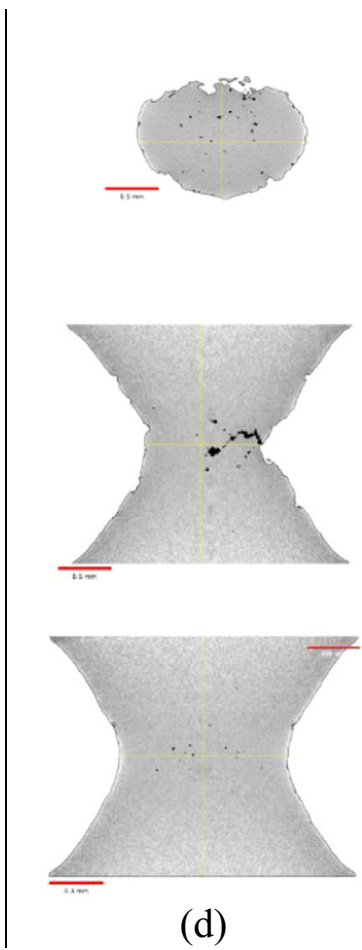

(d)

Fig. 14. X-ray micro-tomography in-situ scans of the hcp-titanium notched specimen subject to uniaxial tension along rolling direction (RD) showing the (TD, ND) view, (RD-TD) view, and (RD, ND) views, respectively corresponding to a notch displacement of: (a) $0.24 \mathrm{~mm}$ (b) $0.73 \mathrm{~mm}$ (c) $1.02 \mathrm{~mm}$, (d) $1.2 \mathrm{~mm}$ 
predicted that for an axisymmetric smooth specimen, damage initiates at the center (see Fig. 11(a)), and shifts toward the outside surface of the specimen for large axial displacements (see Fig. 11(b)-(c)).

\subsection{In-situ XCMT measurements of damage evolution for an axisymmetric notched RD specimen of Ti and comparison with model predictions}

In the previous section, it was shown that Stewart and Cazacu (2011) porous model predicts correctly the level of damage as well as plasticity damage couplings under uniaxial tensile loading of an axisymmetric smooth specimen. The comparison between model and data were done for a fixed level of the imposed axial displacement. Next, the model predictions will be compared to data. Specifically, we will assess the predictive capabilities of the model to capture the influence of the stressstate (stress triaxiality) on damage and its evolution. For this purpose, an uniaxial tension test up to fracture was conducted on a notched axisymmetric RD specimen. The notch radius was of $0.51 \mathrm{~mm}$ while the specimen cross-section radius was of $1.27 \mathrm{~mm}$ (see Fig. 12(a)). The load-displacement curve is shown in Fig. 13 in comparison with the Stewart and Cazacu (2011) F.E. model predictions. Given that the specimen has its axis along an orthotropy axis of the material (RD), only one-eighth of the specimen needs to be meshed. The mesh used consists of 6803 hexahedral elements (ABAQUS C3D8) (see Fig. 12(b)). On Fig. 13 are also shown for comparison the load vs. displacement curves obtained experimentally and predicted by the model for the smooth RD specimen. It is clearly seen that the model describes well that the presence of the notch induces a much softer response as compared to the smooth specimen.

To further provide insights into the porosity evolution in the material and verify the unusual damage characteristics revealed by the model, in-situ XCMT tensile tests on RD specimens of the same notch radius were conducted at Wright Patterson Air Force Laboratory using a Deben CT5000 5 kN in-situ tomograph. Each tomography acquisition was carried out with a cubic voxel size of $3.2516^{3} \mu \mathrm{m}^{3}$ and comprises 700 images of $992 \times 1014$ pixels each. It is to be noted that in order to be able to acquire in-situ XCMT measurements for metallic specimens with this apparatus, the spatial resolution should be larger than that used for the ex-situ measurements done with the VersaXRM-500. The analysis of the XCMT data was done using ImageJ, which is a public domain image processing program developed at the National Institutes of Health (see Rasband, 2014; Schneider et al., 2012; Abramoff et al., 2004).

The rationale for conducting in-situ measurements was to have information on damage evolution in Ti, and validate/ invalidate the trends predicted by the model. Due to the specificity of the in-situ XCMT testing capabilities, the loaddisplacement curve cannot be obtained directly. However, for each XCMT scan, knowing the resolution of the X-ray microscope, it was possible to deduce the displacement between the extremities of the notch. In Fig. 14 are shown views obtained from scans taken at notch displacement of $0.24 \mathrm{~mm}, 0.73 \mathrm{~mm}, 1.02 \mathrm{~mm}$ and $1.20 \mathrm{~mm}$, respectively. To assess the effect of anisotropy on the mechanical response, at each individual level of displacement, the cross-sections in the (TD, ND) plane), in the (RD, TD) plane, and in the (RD, ND) plane are reported (see Fig. 14). Model predictions of the porosity isocontours corresponding to the various cross-sections for several levels of axial displacements are presented in Fig. $15-17$.

A close examination of the scans shown in Fig. 14 for the notched specimen reveals that damage initiates at the surface of the specimen. Thus, the damage evolution predicted by the model (see Fig. 16; Fig. 17) are confirmed experimentally.

Furthermore, it is demonstrated that the location of the zone where damage initiates strongly depends on the specimen geometry. Indeed, the model predicts that for a smooth axisymmetric specimen, damage initiates at the center of the specimen (see Fig. 11(a)), and the level of damage close to failure is very low (see also the comparison between FE predictions and XCMT data for an axial displacement of $5.52 \mathrm{~mm}$ shown in Fig. 10).

On the other hand, for the notched specimen the model predicts that damage initiates at the outer surface of the specimen, and further grow from the outer surface to the center of the specimen (see Fig. 16-17), which corroborates with the in-situ XCMT data of Fig. 14 for the notched specimen.

In other words, for the same global loading (i.e. uniaxial tension), the local stress state, which depends on the specimen geometry is different, which in turn triggers a different damage initiation site, and a markedly different damage evolution.

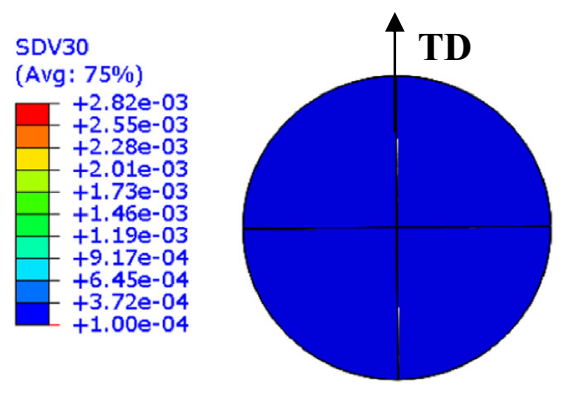

(a)

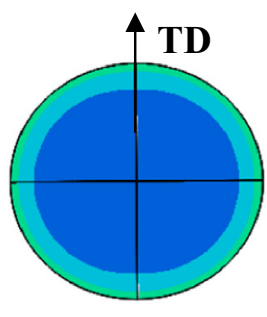

(b)

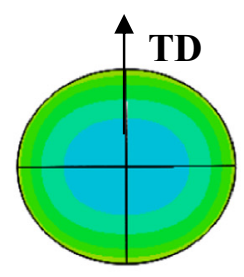

(c)

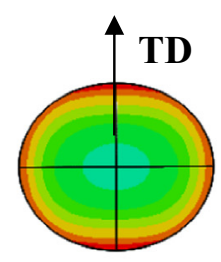

(d)

Fig. 15. F.E. predictions according to the Stewart and Cazacu (2011) model for the void volume fraction isocontours in the (TD, ND) cross-section of an axisymmetric notched specimen of hcp-Ti subjected to uniaxial tension along RD at an axial displacement of: (a) $0.24 \mathrm{~mm}$ (b) $0.73 \mathrm{~mm}$ (c) $1.02 \mathrm{~mm}$ (d) $1.2 \mathrm{~mm}$, respectively. Initial void volume fraction $f_{0}=10^{-4}$. The rolling, transverse, and normal directions are denoted RD, TD, ND, respectively. 

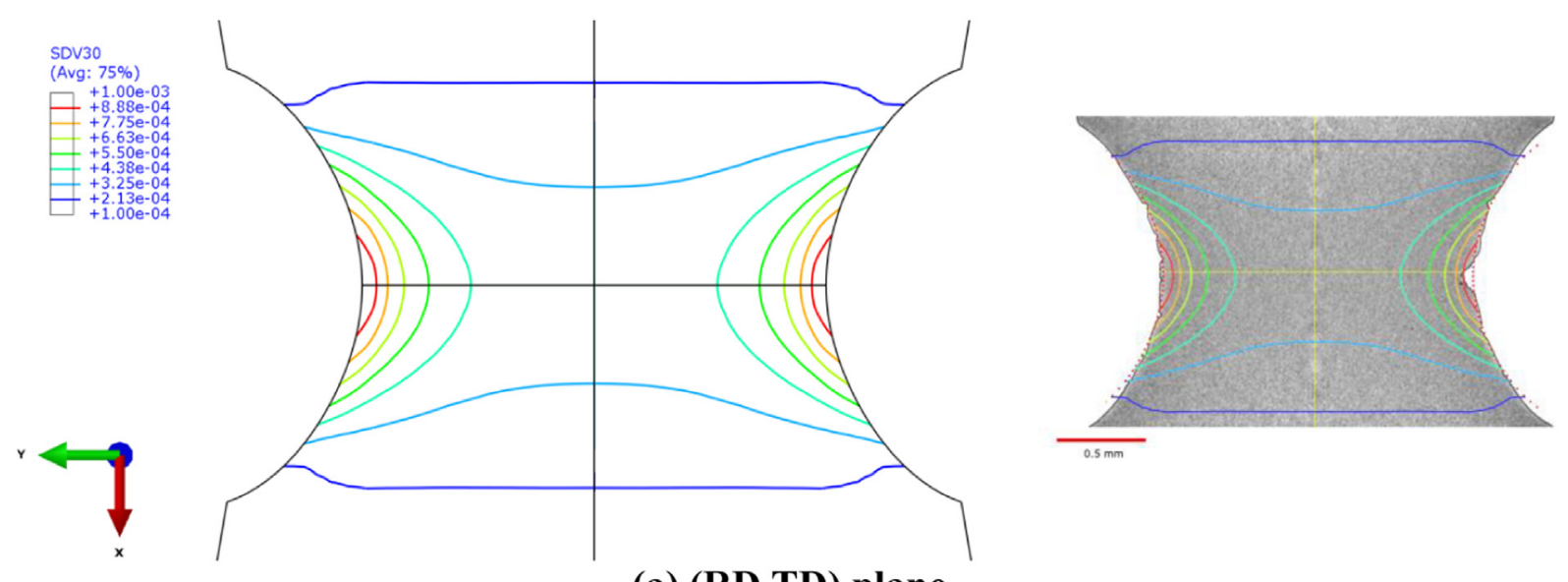

(a) (RD,TD) plane
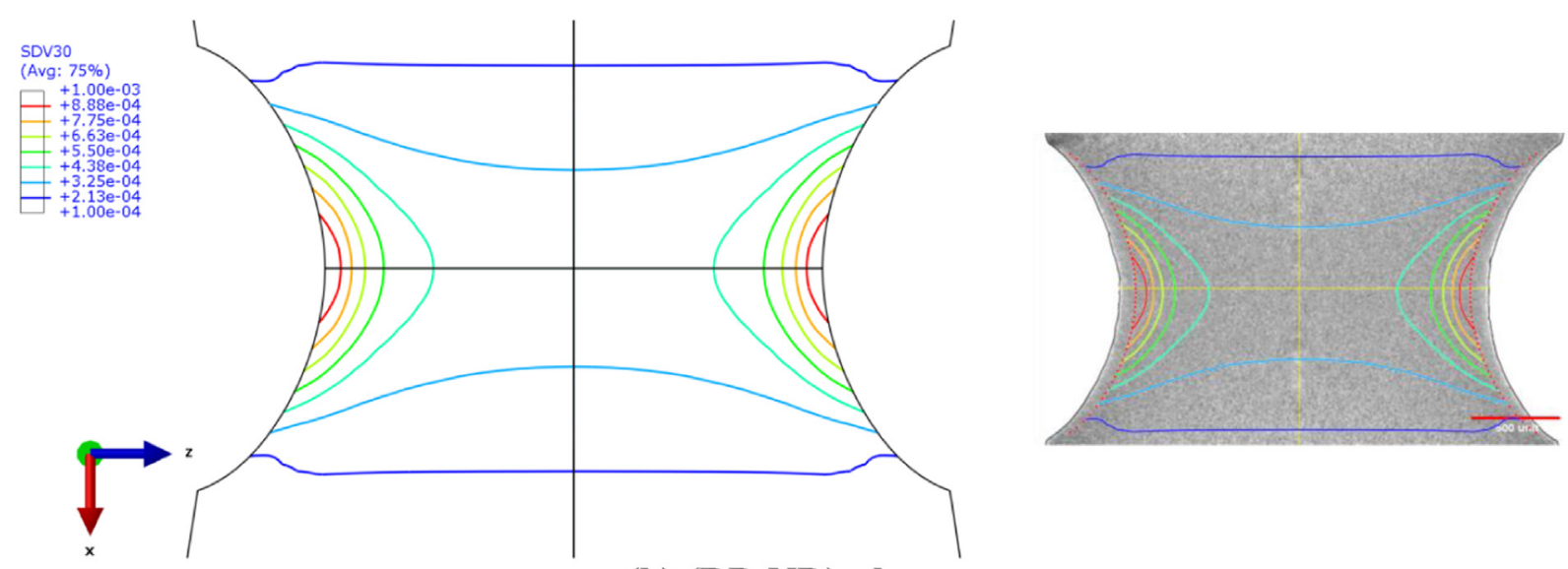

(b) (RD,ND) plane

Fig. 16. Comparison between the F.E. cross-sections and isocontours of void volume fraction of a notched axisymmetric specimen of hcp-titanium subjected to uniaxial tension along RD, according to the Stewart and Cazacu (2011) model and XCMT data for an axial notch displacement of $0.73 \mathrm{~mm}$ : (a) cross-section of the deformed specimen; (b) (RD-TD) section of the deformed specimen; (c) (RD-ND) section of the deformed specimen. The axes $x, y, z$ are along the rolling (RD), transverse (TD), and normal (ND) directions.

It is very important to note that the model predicts that for the specimen subjected to uniaxial tension along RD, damage accumulates differently along ND and TD. The predictions of the isocontours of porosity in the (TD, ND) cross-section corresponding to different levels of notch displacement are shown in Fig. 15. Note that that the void volume fraction is larger along TD (i.e. the small axis of the deformed elliptical cross-section) than along ND (i.e. the long axis of the elliptical crosssection).

Moreover, comparison of the XCMT (RD, TD) sections with the (RD,ND) sections (see Fig. 14), clearly shows that the surface of the specimen along the TD axis is more damaged than the surface along the ND axis. The prediction of the Stewart and Cazacu (2011) porous model corroborates with these experimental observations (see Figs. 16 and 17). To further demonstrate that there is a good agreement between the predictions of the Stewart and Cazacu (2011) model in terms of plasticity-damage couplings in Figs. 16-17 are also superposed on the XCMT scans the F.E. predictions of the specimen profiles (red points) corresponding to notch displacements of $0.73 \mathrm{~mm}$ and $1.2 \mathrm{~mm}$, respectively. Note that the model correctly captures the change in the geometry of the specimen (anisotropy in plastic deformation). Since damage is driven by the plastic deformation, and the plastic anisotropy is correctly described, the model also correctly predicts the location of the zones of maximum damage in each plane.

Because Stewart and Cazacu (2011) plasticity-damage model accounts for both the anisotropic behavior and the tensioncompression asymmetry, the damage evolution predicted by the model captures the main trends revealed by the XCMT scans, namely that damage initiates at the outer surface of the specimen, and further grow from the outer surface to the center of the specimen (see data shown in Fig. 14). To evaluate the importance of the consideration of the tension-compression asymmetry in plastic flow on the local fields, additional FE simulations for the same notched specimen were performed neglecting the tension-compression asymmetry (i.e. in the simulations the tension-compression parameter $k$ is set to zero). As an example, Fig. 18 compares the isocontours of the hydrostatic pressure $(p=-1 / 3 \operatorname{tr}(\sigma))$ in the (RD-TD) 

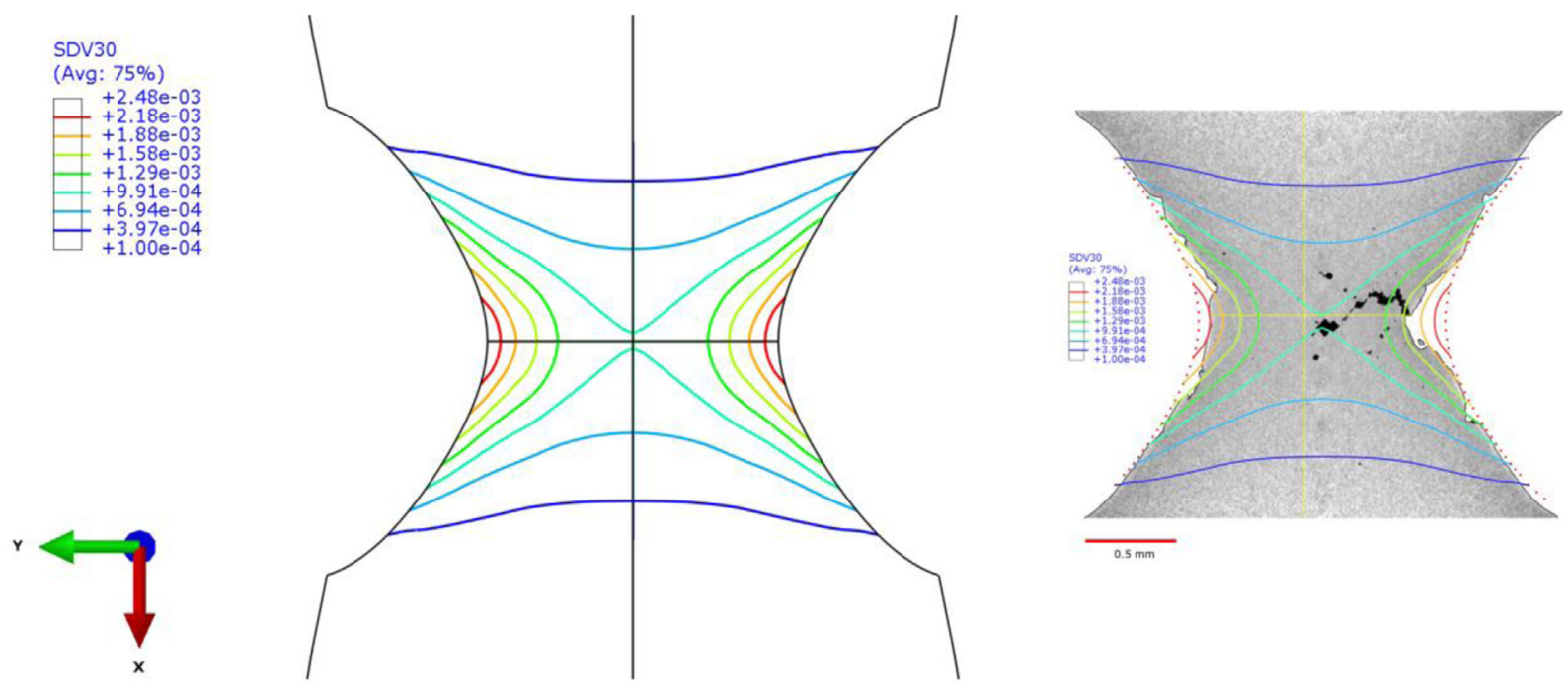

(a) (RD,TD) plane
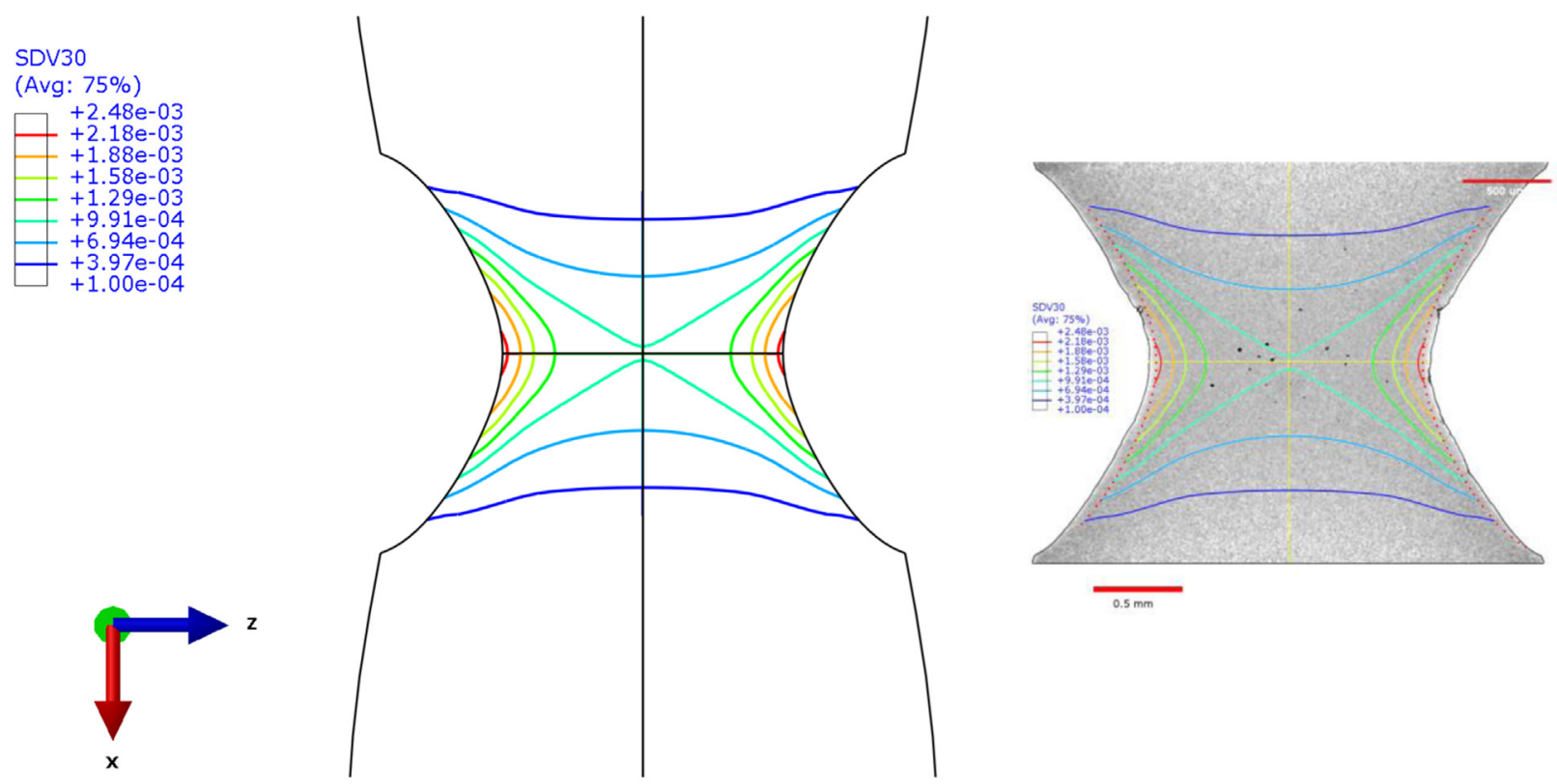

(b) (RD,ND) plane

Fig. 17. Comparison between the F.E. cross-sections and isocontours of void volume fraction of a notched axisymmetric specimen of hcp-titanium subjected to uniaxial tension along RD, according to the Stewart and Cazacu (2011) model and XCMT data for an axial notch displacement of 1.2 mm: (a) crosssection of the deformed specimen; (b) (RD-TD) section of the deformed specimen; (c) (RD-ND) section of the deformed specimen. The axes $x, y, z$ are along the rolling (RD), transverse (TD), and normal (ND) directions.

section of the notched specimen at axial notch displacement of $0.73 \mathrm{~mm}$, and $1.2 \mathrm{~mm}$, respectively according with the anisotropic model with no tension-compression symmetry $(k=0)$ and the Stewart and Cazacu (2011) model. Note that the distribution of the hydrostatic pressure is totally different. If the tension-compression is neglected, the maximum mean stress (i.e. the minimum hydrostatic pressure) occurs in the center of the specimen, while if the model accounts for the tension compression asymmetry, the maximum mean stress is located along the boundary surface of the specimen. To pursue this analysis, in Fig.19 are plotted the isocontours of the third-invariant of the stress deviator for the same axial displacements. If the tension-compression asymmetry is neglected, locally, the third invariant of the stress deviator is always positive. However, if the model accounts for both anisotropy and tension-compression asymmetry, the third-invariant $\mathrm{J}_{3}$ is negative close to the outside surface of the specimen and positive at the center of the specimen. Given that the strong 


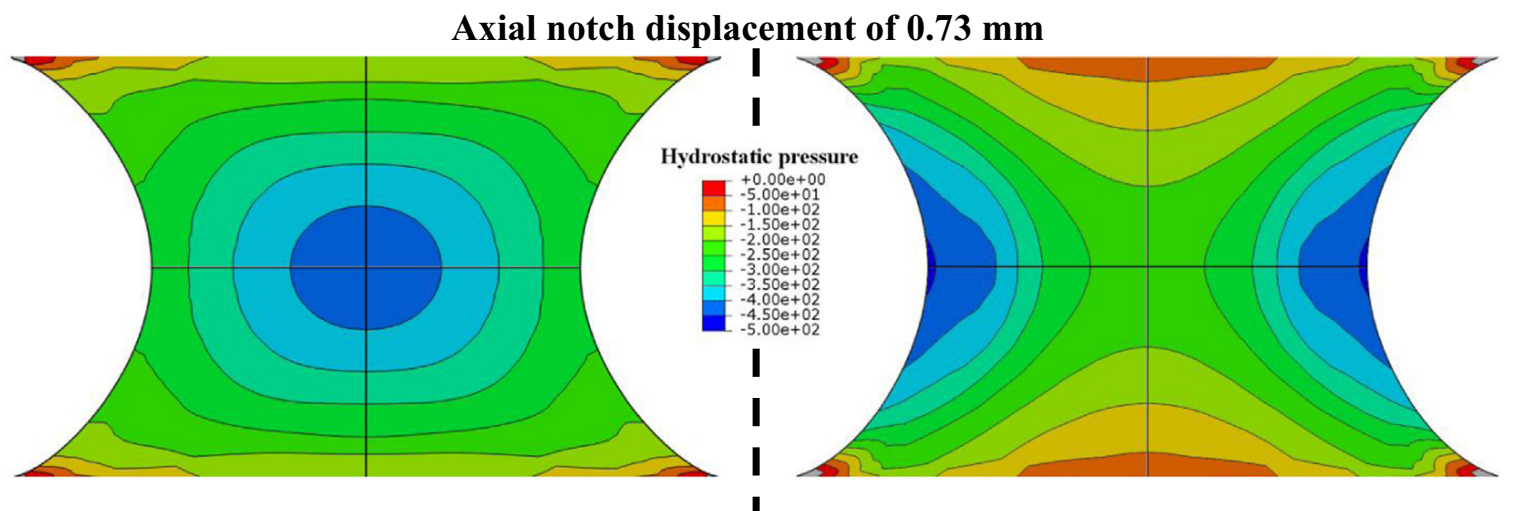

cement of $1.2 \mathrm{~mm}$

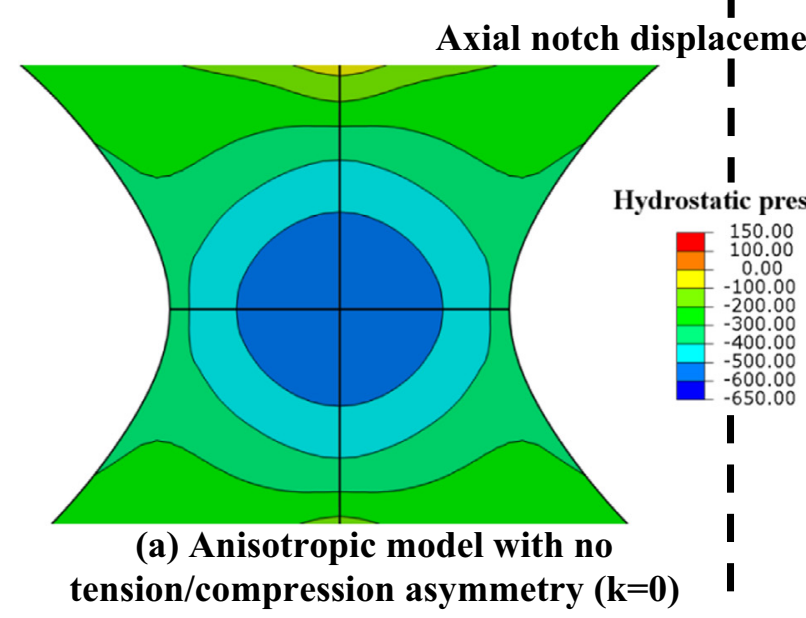

(b) Present model accounting for
anisotropy and tension-compression
asymmetry

Fig. 18. Importance of accounting for tension-compression asymmetry in plastic behavior of hcp-titanium: comparison of the isocontours of the hydrostatic pressure $(p=-1 / 3 \operatorname{tr}(\sigma))$ in the (RD-TD) section of the notched specimen for axial notch displacement of $0.73 \mathrm{~mm}$, and $1.2 \mathrm{~mm}$, respectively according with: (a) anisotropic model with no tension-compression asymmetry $(k=0)$, (b) Stewart and Cazacu (2011) model which accounts for both anisotropy and tension-compression asymmetry. Note that if the tension-compression asymmetry in plastic deformation is accounted for, the local distribution of the hydrostatic pressure is totally different.

coupling between the mean stress and the third-invariant $\mathrm{J}_{3}$ on the response of the porous material (see the Eq. (3) and the yield surface of the porous hcp Ti shown in Fig. 5), the isocontours of the void volume fraction are completely different depending on whether the tension-compression is accounted in the plasticity-damage model or not (see Fig. 20). If the tension-compression is neglected, the maximum void volume fraction is located at the center of the specimen, while if the model accounts for the tension-compression asymmetry, the zone of maximum damage shifts toward the outside surface.

\section{Summary and conclusions}

In this paper, results of an experimental study on the room-temperature quasi-static plastic deformation and damage of a high-purity, polycrystalline, HCP-titanium material were presented. To quantify the plastic anisotropy and the tensioncompression symmetry of the material, first monotonic uniaxial compression and tension tests were carried out. This study shows that for Ti materials the type of material symmetry can be assed correctly only if $r$-values measurements are also performed. While the true stress-true strain curves in different in-plane orientations are very close (see Fig. 2-3), the material exhibits a strong anisotropy in Lankford coefficients with the $r$-value along RD being much larger than in the 45 and TD directions. There is also a marked difference between the stress-strain response in the normal plate direction and the in-plane directions. On the basis of all the test results it can be concluded that the material is orthotropic. Furthermore, irrespective of the loading orientation, the material displays strength-differential effects (harder in compression than in tension). Digital image correlation techniques have been used to determine the strain fields (axial and width strains) in the gage zone. To characterize the evolution of anisotropy with accumulated plastic strain, for each specimen orientation, Lankford coefficients ( $r$-values) were determined for several levels of plastic strain. Irrespective of the strain level, the largest $r$-value is along RD.

Stewart and Cazacu (2011) anisotropic elastic/plastic damage model was applied to describe the behavior of the material 

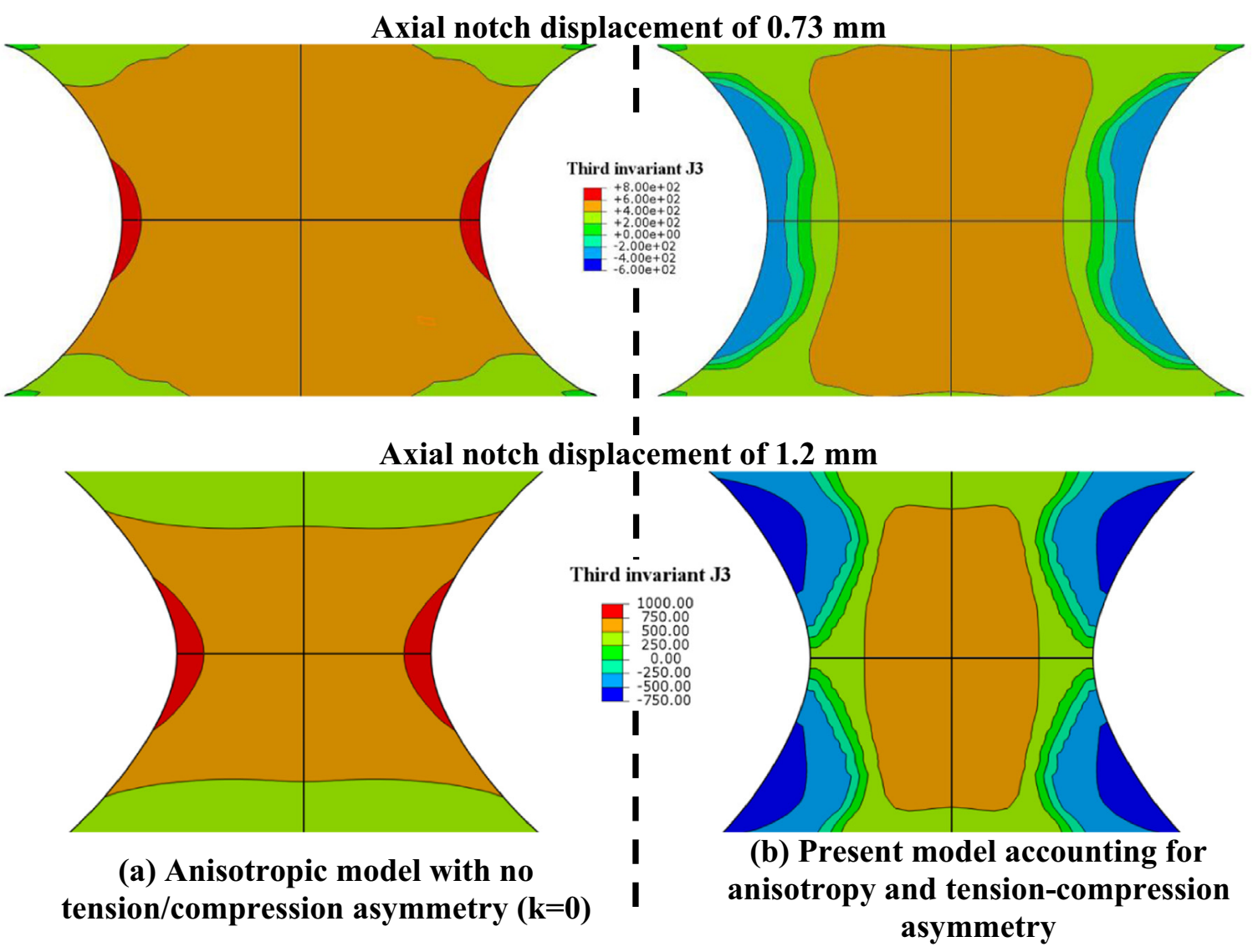

Fig. 19. Importance of accounting the tension-compression asymmetry of the plastic behavior of an hcp-titanium. Comparison of the isocontours of the third-invariant of the stress deviator $\mathrm{J}_{3}$ in the (RD-TD) section of the notched specimen for an axial notch displacement of $0.73 \mathrm{~mm}$ and $1.2 \mathrm{~mm}$, respectively according with: (a) anisotropic model with no tension-compression asymmetry $(k=0)$, (b) Stewart and Cazacu (2011) model which accounts for both anisotropy and tension-compression asymmetry. Note that only if the the tension-compression asymmetry in plastic deformation is neglected, the third-invariant is always positive, otherwise the local distribution is completely different.

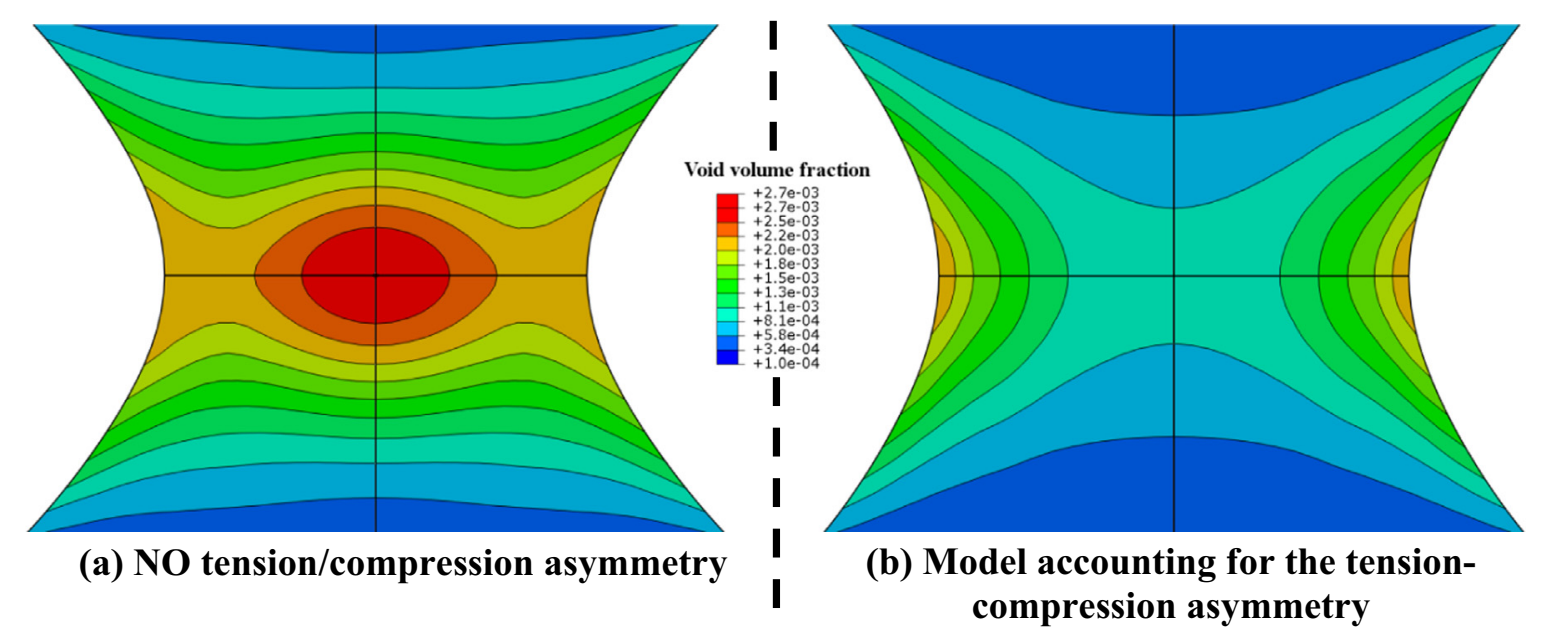

Fig. 20. Importance of accounting the tension-compression asymmetry of the plastic behavior of an hcp-titanium. Comparison of the isocontours of the void volume fraction in the (RD-TD) section of the notched specimen for an axial notch displacement of $1.2 \mathrm{~mm}$, respectively according with: (a) anisotropic model with no tension-compression asymmetry $(k=0)$, (b) Stewart and Cazacu (2011) model which accounts for both anisotropy and tension-compression asymmetry. 
studied. All the parameters involved in the Stewart and Cazacu (2011) model have a clear physical significance, being related to plastic properties. Specifically, these parameters were identified based on the experimental data obtained in a few mechanical characterization tests (uniaxial tension tests on specimens of rectangular cross-section, and uniaxial compression tests).

In addition to the mechanical characterization tests on flat tensile specimens, uniaxial tensile tests on cylindrical specimens with circular cross-section were also conducted. To further the understanding of the plasticity-damage couplings of the material, and assess the predictive capabilities of the model, both ex-situ and in-situ XCMT measurements were done on the specimen oriented along the rolling direction. Specifically, the F.E. predictions obtained using the same set of parameters previously identified were compared with the measured final cross-sections and XCMT measurements of porosity for both smooth and notched specimens.

The XCMT measurements on axisymmetric smooth specimens reveal the drastic difference in damage evolution between the hcp-Ti specimen and a copper material for which the plastic deformation is governed by von Mises criterion. For the same axial displacement and similar levels of plastic deformation, the copper material is much damaged while the titanium material shows very little damage. While a large hole/crack is seen in the middle of the copper specimen, almost no damage is observed in the hcp-Ti specimen. The data indicate that there is a very strong correlation between the characteristics of the plastic deformation and the rate of damage growth. This is contrary with the generally held belief that damage evolution in Ti can be predicted using models and damage evolution laws originally developed for materials for which the plastic behavior is governed by either von Mises or Hill (1948) criterion.

It was shown that using a model that accounts for the specificities of the plastic deformation in titanium both the anisotropy of plastic deformation and damage can be captured. Irrespective of the loading orientation the final geometry of the specimens was correctly predicted. Specifically, it is predicted that for a smooth axisymmetric specimen subject to uniaxial tension, damage initiates at the center of the specimen and is diffuse; the level of damage close to failure is very low. On the other hand, for a notched specimen subject to uniaxial tension the model predicts that damage initiates at the outer surface of the specimen, and further grows from the outer surface to the center of the specimen, which corroborates with the in-situ XCMT data.

\section{Appendix A. : Components of the 4th order anisotropic tensor B}

The parameter $\mathrm{h}$ involved in the expression of the criterion describes damage evolution. Its expression (see Eq. (7)-(9)) depends on the components of the tensor $\mathbf{B}$, the inverse of $\mathbf{C}=\mathbf{L K}$ (see the representation of $\mathbf{L}$ and $\mathbf{K}$ given by Eq. (5) and Eq. (10), respectively). Thus, given the values of the anisotropy coefficients $\mathrm{L}_{\mathrm{ij}}$, in order to calculate h, one needs to know the expressions of the components of $\mathbf{B}$.

The components of the tensor $\mathbf{B}$ (Voigt notations) in terms of $\mathrm{C}_{\mathrm{ij}}$ are given by:

$$
\begin{aligned}
& B_{12}=\frac{1}{3} \cdot \frac{2\left(C_{32}-C_{12}\right)+\left(C_{31}-C_{11}\right)}{\left(C_{21}-C_{11}\right)\left(C_{32}-C_{12}\right)-\left(C_{22}-C_{12}\right)\left(C_{31}-C_{11}\right)} \\
& B_{13}=\frac{1}{3} \cdot \frac{\left(C_{11}-C_{21}\right)+2\left(-C_{22}+C_{12}\right)}{\left(C_{21}-C_{11}\right)\left(C_{32}-C_{12}\right)-\left(C_{22}-C_{12}\right)\left(C_{31}-C_{11}\right)} \\
& B_{11}=-\left(B_{12}+B_{13}\right) \\
& B_{21}=B_{12} \\
& B_{23}=\frac{1}{3} \cdot \frac{2\left(C_{11}-C_{21}\right)+\left(C_{12}-C_{22}\right)}{\left(C_{11}-C_{21}\right)\left(C_{32}-C_{22}\right)-\left(-C_{22}+C_{12}\right)\left(C_{31}-C_{21}\right)} \\
& B_{22}=-\left(B_{21}+B_{23}\right) \\
& B_{31}=B_{13} \\
& B_{32}=B_{23} \\
& B_{33}=-\left(B_{31}+B_{23}\right) \\
& B_{44}=\frac{1}{C_{44}}, \quad B_{55}=\frac{1}{C_{55}}, \quad B_{66}=\frac{1}{C_{66}} .
\end{aligned}
$$

\section{Appendix B. Identification of the coefficients of the Stewart and Cazacu (2011) criterion}

All model parameters have a clear physical significance, being expressible in terms of the anisotropy coefficients (components of the tensor $\mathbf{L}$ ) and the strength differential parameter k. The anisotropy coefficients $L_{i j}$, with $i, j=1 \ldots 3$ and $L_{44}$ can be determined from the experimental normalized tensile flow stress values $\tilde{\sigma}_{\theta}^{T}=\sigma_{\theta}^{T} / \sigma_{x}^{T}$ and compressive flow stresses $\tilde{\sigma}_{\theta}^{C}=\sigma_{\theta}^{C} / \sigma_{x}^{T}$ and Lankford coefficients $r_{\theta}(\theta$ represents the angle between the uniaxial loading direction and the RD direction) reported in Section 3. The theoretical expressions of $\tilde{\sigma}_{\theta}^{T}=\sigma_{\theta}^{T} / \sigma_{x}^{T} \tilde{\sigma}_{\theta}^{C}=\sigma_{\theta}^{C} / \sigma_{x}^{T}$ necessary for writing the cost function to be used in the minimization procedure are provided in the following. 
Note that for in-plane loadings (only $\sigma_{\mathrm{xx}}, \sigma_{\mathrm{yy}}, \sigma_{x y}$ non-zero), the only non-zero components of the transformed stress tensor $\widehat{\boldsymbol{\sigma}}=\mathrm{L}: \boldsymbol{\sigma}^{\prime}$ (see Eq. (4)) are:

$$
\widehat{\sigma}_{\mathrm{xx}}=\Phi_{1} \sigma_{\mathrm{xx}}+\Psi_{1} \sigma_{\mathrm{yy}}, \quad \widehat{\sigma}_{y y}=\Phi_{2} \sigma_{\mathrm{xx}}+\Psi_{2} \sigma_{y y}, \quad \widehat{\sigma}_{z z}=\Phi_{3} \sigma_{\mathrm{xx}}+\Psi_{3} \sigma_{y y} \text { and } \widehat{\sigma}_{\mathrm{xy}}=\mathrm{L}_{44} \sigma_{\mathrm{xy}},
$$

with

$$
\begin{aligned}
& \Phi_{1}=\left(2 L_{11}-L_{12}-L_{13}\right) / 3, \quad \Phi_{2}=\left(2 L_{12}-L_{22}-L_{23}\right) / 3, \quad \Phi_{3}=\left(2 L_{13}-L_{23}-L_{33}\right) / 3, \\
& \Psi_{1}=\left(-L_{11}+2 L_{12}-L_{13}\right) / 3, \Psi_{2}=\left(-L_{12}+2 L_{22}-L_{23}\right) / 3, \quad \Psi_{3}=\left(-L_{13}+2 L_{23}-L_{33}\right) / 3 .
\end{aligned}
$$

Hence, the principal values of the transformed tensor $\widehat{\sigma}$ are given by

$$
\widehat{\sigma}_{1,2}=\left(\widehat{\sigma}_{\mathrm{xx}}+\widehat{\sigma}_{\mathrm{yy}} \pm \sqrt{\left(\widehat{\sigma}_{\mathrm{xx}}-\widehat{\sigma}_{\mathrm{yy}}\right)^{2}+4 \widehat{\sigma}_{\mathrm{xy}}^{2}}\right) / 2 \text { and } \widehat{\sigma}_{3}=\widehat{\sigma}_{\mathrm{zz}}
$$

Using Eq. (B.1) and (B.2), it follows that according to the Cazacu et al. (2006) criterion (Eq. (14))

$$
\sigma_{\theta}^{T}=\sigma_{x}^{T} \sqrt{\frac{\left(\left|\Phi_{1}\right|-k \Phi_{1}\right)^{2}+\left(\left|\Phi_{2}\right|-k \Phi_{2}\right)^{2}+\left(\left|\Phi_{3}\right|-k \Phi_{3}\right)^{2}}{\left(\left|\mathrm{~A}_{1}(\theta)\right|-k\left|\mathrm{~A}_{1}(\theta)\right|\right)^{2}+\left(\left|\mathrm{A}_{2}(\theta)\right|-k\left|\mathrm{~A}_{2}(\theta)\right|\right)^{2}+\left(\left|\mathrm{A}_{3}(\theta)\right|-k\left|\mathrm{~A}_{3}(\theta)\right|\right)^{2}}}
$$

where $\sigma_{x}^{T}$ is the tensile yield stress in the rolling direction (i.e. for $\theta=0^{\circ}$ ) and

$\mathrm{A}_{1,2}(\theta)=\cos ^{2} \theta\left(\Phi_{1}+\Phi_{2}\right) / 2+\sin ^{2} \theta\left(\Psi_{1}+\Psi_{2}\right) / 2 \pm \sqrt{\Delta}, \quad A_{3}=\Phi_{3} \cos ^{2} \theta+\Psi_{3} \sin ^{2} \theta$ with

$$
\Delta=\sqrt{\left(\cos ^{2} \theta\left(\Phi_{1}-\Phi_{2}\right) / 2+\sin ^{2} \theta\left(\Psi_{1}-\Psi_{2}\right) / 2\right)^{2}+\mathrm{L}_{44}^{2} \sin ^{2} \theta \cos ^{2} \theta} .
$$

For uniaxial compressive loadings in the plane of the plate,

$$
\sigma_{\theta}^{C}=\sigma_{x}^{T} \sqrt{\frac{\left(\left|\Phi_{1}\right|-k \Phi_{1}\right)^{2}+\left(\left|\Phi_{2}\right|-k \Phi_{2}\right)^{2}+\left(\left|\Phi_{3}\right|-k \Phi_{3}\right)^{2}}{\left(\left|\mathrm{~A}_{1}(\theta)\right|+k\left|\mathrm{~A}_{1}(\theta)\right|\right)^{2}+\left(\left|\mathrm{A}_{2}(\theta)\right|+k\left|\mathrm{~A}_{2}(\theta)\right|\right)^{2}+\left(\left|\mathrm{A}_{3}(\theta)\right|+k\left|\mathrm{~A}_{3}(\theta)\right|\right)^{2}}}
$$

The plastic strain ratio, $r_{\theta}$ under uniaxial tensile loading in a direction at angle $\theta$ with the rolling direction in the plane (xy) is:

$$
r_{\theta}=-\frac{\sin ^{2} \theta \frac{\partial \tilde{\sigma}_{e}}{\partial \sigma_{\mathrm{xx}}}-\sin (2 \theta) \frac{\partial \tilde{\sigma}_{e}}{\partial \sigma_{\mathrm{xy}}}+\cos ^{2} \theta \frac{\partial \tilde{\sigma}_{e}}{\partial \sigma_{\mathrm{yy}}}}{\frac{\partial \tilde{\sigma}_{e}}{\partial \sigma_{\mathrm{xx}}}+\frac{\partial \tilde{\sigma}_{e}}{\partial \sigma_{\mathrm{yy}}}}
$$

where $\tilde{\sigma}_{e}=\hat{m} \sqrt{\sum^{3}\left(\left|\widehat{\sigma}_{i}\right|-k \widehat{\sigma}_{i}\right)^{2}}$ and $\hat{m}$ given by Eq. (6).

Note that for ${ }^{1}$ uniaxial loadings in the normal direction of the plate, the respective yield stresses are:

$$
\begin{aligned}
& \sigma_{z}^{C}=\sigma_{x}^{T} \sqrt{\frac{\left(\left|\Phi_{1}\right|-k \Phi_{1}\right)^{2}+\left(\left|\Phi_{2}\right|-k \Phi_{2}\right)^{2}+\left(\left|\Phi_{3}\right|-k \Phi_{3}\right)^{2}}{\left(\left|\Omega_{1}\right|+k\left|\Omega_{1}\right|\right)^{2}+\left(\left|\Omega_{2}\right|+k\left|\Omega_{2}\right|\right)^{2}+\left(\left|\Omega_{3}\right|+k\left|\Omega_{3}\right|\right)^{2}}} \\
& \sigma_{z}^{T}=\sigma_{x}^{T} \sqrt{\frac{\left(\left|\Phi_{1}\right|-k \Phi_{1}\right)^{2}+\left(\left|\Phi_{2}\right|-k \Phi_{2}\right)^{2}+\left(\left|\Phi_{3}\right|-k \Phi_{3}\right)^{2}}{\left(\left|\Omega_{1}\right|-k\left|\Omega_{1}\right|\right)^{2}+\left(\left|\Omega_{2}\right|-k\left|\Omega_{2}\right|\right)^{2}+\left(\left|\Omega_{3}\right|-k\left|\Omega_{3}\right|\right)^{2}}},
\end{aligned}
$$

where $\Omega_{1}=\left(L_{11}+L_{12}-2 L_{13}\right) / 3, \Omega_{2}=\left(L_{12}+L_{22}-2 L_{23}\right) / 3, \Omega_{3}=\left(L_{13}+L_{23}-2 L_{33}\right)$.

In conclusion, using eqs. (B.1)-(B.7), the model parameters can be determined by minimizing an error function of the form

$$
\mathrm{F}=\sum_{i}^{n} \eta_{i}\left(1-\frac{\left(\sigma_{\theta}^{T}\right)_{i}^{\mathrm{th}}}{\left(\sigma_{\theta}^{T}\right)_{i}^{\text {data }}}\right)^{2}+\sum_{j}^{m} \gamma_{j}\left(1-\frac{\mathrm{r}_{j}^{\mathrm{th}}}{\mathrm{r}_{j}^{\text {data }}}\right)^{2}+\sum_{l}^{p} \alpha_{l}\left(1-\frac{\left(\sigma_{\theta}^{C}\right)_{i}^{\mathrm{th}}}{\left(\sigma_{\theta}^{C}\right)_{i}^{\text {data }}}\right)^{2}
$$

In the above equation, " $n$ " (respectively, $m$ and $p$ ) represents the number of experimental directional flow stresses (respectively, $r$-ratios) available, the superscript indicates whether the respective value is experimental or calculated using the expressions (B.1)-(B.8) while $\eta_{i}, \gamma_{j}$, and $\alpha_{l}$ are weight factors. For the Ti material studied all the weight factors were set to unity. 


\section{References}

ABAQUS, 2009. User's Manual for Version 6.8. vol. I-V. Dassault Systemes Simulia Corp., Providence, RI.

Abramoff, M.D., Magalhaes, P.J., Ram, S.J., 2004. Image Processing with ImageJ. Biophotonics Int. 11 (7), $36-42$.

Argon, A.S., Im, J., Safoglu, R., 1975. Cavity formation from inclusions in ductile fracture. Metall. Trans. A 6 (4), $825-837$.

Banerjee, D., Williams, J.C., 2013. Perspectives on titanium science and technology. Acta Mater. 61, 844-879.

Barlat, F., Richmond, O., 1987. Prediction of tricomponent plane Stress yield surfaces and associated flow and failure behavior of strongly textured F.C.C. polycrystalline sheets. Mater. Sci. Eng. 95, 15-29.

Barlat, F., Cazacu, O., Zyczkowski, M., Banabic, D., Yoon, J.W., 2004. Yield surface plasticity and anisotropy in sheet metals. In: Raabe, D., Roters, F., Barlat, F. Chen, L.-Q. (Eds.), Continuum Scale Simulation of Engineering Materials, Fundamentals - Microstructures - Process Applications, vol. 145. Wiley-VCH Verlag Berlin GmbH, Weinheim.

Benhenni, N., Bouvier, S., Brenner, R., Chauveau, T., Bacroix, B., 2013. Micromechanical modelling of monotonic loading of CP-a titanium: correlation between macroscopic and microscopic behavior. Mater. Sci. Eng. A 573, 222.

Bouvier, S., Benmhenni, N., Tirry, W., Gregory, F., Nixon, M.E., Cazacu, O., Rabet, L., 2012. Hardening in relation with microstructure evolution of high-purity alpha-titanium deformed under monotonic and cyclic loading at room temperature. Mater. Sci. Eng. A 535, 12-21.

Cazacu, O., Barlat, F., 2004. A criterion for description of anisotropy and yield differential effects in pressure-insensitive metals. Int. J. Plast. 20 (11), 2027-2045.

Cazacu, O., Stewart, J.B., 2009. Analytic plastic potential for porous aggregates with matrix exhibiting tension-compression asymmetry. J. Mech. Phys. Solids $57(2), 325-341$.

Cazacu, O., Plunkett, B., Barlat, F., 2006. Orthotropic yield criterion for hexagonal closed packed metals. Int. J. Plast. 22 (7), $1171-1194$.

Cazacu, O., Revil-Baudard, B., Lebensohn, R.A., Garajeu, M., 2013. On the combined effects of pressure and third-invariant on yielding of porous solids with von Mises matrix. J. Appl. Mech. 80 (6), 064501-064501-5.

Cazacu, O., Revil-Baudard, B., Chandola, N., Kondo, D., 2014. Analytical criterion for porous solids with Tresca matrix accounting for combined effects of the mean stress and third-invariant of the stress deviator. Int. J. Solids Struct. 51, 861-874.

Chu, C.C., Needleman, A., 1980. Void nucleation effects in biaxially stretched sheets. J. Eng. Mater. Technol. Trans. ASME 102 (3), $249-256$.

Chun, Y.B., Yu, S.L., Semiatin, S.L., Hwang, S.K., 2005. Effect of deformation twinning on microstructure and texture evolution during cold rolling of CPtitanium. Mater. Sci. Eng. A 398, 209-219.

Gilles, G., Habraken, A.M., Cazacu, O., Balan, T., Duchêne, L., 2013. Experimental and numerical study of TA6V mechanical behavior under different quasistatic strain paths at room temperature. AIP Conf. Proc. 1532, 651-656.

Gilles, G., Hammami, W., Libertiaux, V., Cazacu, O., Yoon, J.H., Kuwabara, T., Habraken, A.M., Duchêne, L., 2011. Experimental characterization and elastoplastic modeling of the quasi-static mechanical response of TA-6 V at room temperature. Int. J. Solids Struct. 48, $1277-1289$.

Green, A.E., Naghdi, P.M., 1965. A general theory of an elastic-plastic continuum. Arch. Ration. Mech. Anal. 18, $251-281$.

Gurland, J., 1972. Observations on the fracture of cementite particles in a spheroidized 1.05\% C steel deformed at room temperature. Acta Metall. 20 (5), $735-741$.

Gurson, A.L., 1977. Continuum theory of ductile rupture by void nucleation and growth: Part I: yield criteria and flow rules for porous ductile media. J. Eng. Mater. Technol. Trans. ASME Ser. H 99, 2-15.

Gurson, A., 1975. Plastic Flow and Fracture Behaviour of Ductile Materials Incorporating Void Nucleation, Growth, and Interaction. (Ph.D. thesis), Brown University, Rhode Island, USA.

Hill, R., 1948. A theory of the yielding and plastic flow of anisotropic materials. Proc. R. Soc. Lond. A 193, $281-297$.

Hill, R., 1967. The essential structure of constitutive laws for metal composites and polycrystals. J. Mech. Phys. Solids 15, 79-95.

Huez, J., Feugas, X., Helbert, A.L., Guillot, I., Clavel, M., 1998. Damage process in Commercially Pure $\alpha$-Titanium Alloy without (Ti40) and with (Ti40-H) Hydrides. Metall. Mater. Trans. A. 29, 1615.

Knezevic, M., Lebensohn, R.A., Cazacu, O., Revil-Baudard, B., Proust, G., Vogel, S.C., Nixon, M.E., 2013. Modeling bending of $\alpha$-titanium with embedded polycrystal plasticity in implicit finite elements. Mater. Sci. Eng. A 564, 116-126.

Kuwabara T., Katami C., Kikuchi M., Shindo T., Ohwue T., 2001. Cup drawing of pure titanium sheet-finite element analysis and experimental validation. In: Proceedings of the 7th International Conference on Numerical Methods in Industrial Forming Processes. 18-20 June, Toyohashi, Japan, pp. 781-787.

Lebensohn, R.A., Cazacu, O., 2012. Effect of single-crystal plastic deformation mechanisms on the dilatational plastic response of porous polycrystals. Int. J. Solids Struct. 49, 3838-3852.

Mandel J., 1972. Plasticité Classique et Viscoplasticité. CISM Courses and Lectures, International Center for Mechanical Sciences, Springer-Verlag, Wien-New York, Vol. 97.

Nixon, M.E., Cazacu, O., Lebensohn, R.A., 2010b. Anisotropic response of high-purity $\alpha$-titanium: experimental characterization and constitutive modeling. Int. J. Plast. 26, 510-532.

Nixon, M.E., Lebensohn, R.A., Cazacu, O., Liu, C., 2010a. Experimental and finite-element analysis of the anisotropic response of high-purity $\alpha$-titanium in bending. Acta Mater. 58, 5759-5767.

Rasband, W.S., ImageJ, U.S. National Institutes of Health, Bethesda, Maryland, USA, 1997-2014. 〈http://imagej.nih.gov/ij/〉.

Revil-Baudard, B., Cazacu, O., 2013. On the effect of the matrix tension-compression asymmetry on damage evolution in porous plastic solids. Eur. J. Mech. - A/Solids 37, 35-44.

Revil-Baudard, B., Cazacu, O., Chandola, N., Barlat, F., 2014. Correlation between Swift effects and tension-compression asymmetry in various polycrystalline materials. J. Mech. Phys. Solids 70, 104-115.

Revil-Baudard, B., Cazacu, O., Flater, P., Kleiser, G., 2015. Plastic deformation of high-purity $\alpha$-titanium: model development and validation using the Taylor cylinder impact test. Mech. Mater. 80, 264-275. (2015).

Rice, J.R., Tracey, D.M., 1969. On the ductile enlargement of voids in triaxial stress fields. J. Mech. Phys. Solids 17, $201-217$.

Salem, A.A., Kalidindi, S.R., Doherty, R.D., 2003. Strain hardening of titanium: role of deformation twinning. Acta Mater. 51 , $4225-4237$.

Schneider, C.A., Rasband, W.S., Eliceiri, K.W., 2012. NIH Image to ImageJ: 25 years of image analysis. Nat. Methods 9, 671-675.

Stewart, J.B., Cazacu, O., 2011. Analytical yield criterion for an anisotropic material containing spherical voids and exhibiting tension-compression asymmetry. Int. J. Solids Struct. 48 (2), 357-373. 